\title{
VISUAL AND NEAR-INFRARED IMAGING OF ULTRALUMINOUS INFRARED GALAXIES: THE IRAS 2 Jy SAMPLE
}

\author{
T. W. Murphy, JR., L. ARmus, K. MatThews, AND B. T. SoIfer \\ Palomar Observatory, California Institute of Technology, 320-47, Pasadena, California 91125 \\ Electronic mail: tmurphy@mop.caltech.edu \\ J. M. MazZarella AND D. L. ShUPE \\ Infrared Processing \& Analysis Center, California Institute of Technology, Jet Propulsion Laboratory, 100-22, Pasadena, \\ California 91125 \\ M. A. STRAuSS \\ Institute for Advanced Study, School of Natural Sciences, Princeton, New Jersey 08540

\section{G. NEUGEBAUER} \\ Palomar Observatory, California Institute of Technology, 320-47, Pasadena, California 91125 \\ Received 1995 August 7; revised 1995 December 11
}

\begin{abstract}
New near-infrared and visual images at $2.2 \mu \mathrm{m}$ and $6550 \AA$ are presented for 46 galaxies having infrared luminosities of $L_{\mathrm{IR}}>8.5 \times 10^{11} L_{\odot}, 60 \mu \mathrm{m}$ flux densities greater than $1.94 \mathrm{Jy}$, and declinations greater than $-35^{\circ}$. These galaxies make up a significant fraction of a complete, northern hemisphere sample of ultraluminous infrared galaxies. Visual and/or near-infrared imaging data now exist for 56 ultraluminous infrared galaxies out to nearly $50000 \mathrm{~km} \mathrm{~s}^{-1}$. Of these 56 galaxies, $53(95 \%)$ show evidence for current or past interactions. Among these systems, there are a large variety of visual morphologies, including strongly interacting pairs with apparent tidal tails, as well as single, distorted galaxies with close double nuclei. There are three galaxies which, to the limits of the imaging data, do not appear to have suffered a recent interaction or merger. Approximately $47 \%$ (25/53) of the interacting systems have double nuclei, with projected nuclear separations ranging from 0.3 to $48 \mathrm{kpc}$. Seven systems have nuclear separations larger than $10 \mathrm{kpc}$. If the 53 interacting galaxies are viewed as stages in the evolution of pairs of interacting spiral galaxies to a single, luminous AGN or starburst, the present imaging data can be used to estimate the lifetime of the bright infrared phase. Including only those sample galaxies with morphological evidence for interactions, we calculate a lower and an upper limit to the lifetime of the ultraluminous infrared phase of the sample as a whole to be $2 \times 10^{8}$ and $2 \times 10^{9}$ yr, respectively. Comparison of these dynamical estimates to models of the mergers of gas-rich galaxies and the rates at which fuel is exhausted by starbursts or AGN suggests the lifetime of the ultraluminous phase lies much closer to the smaller of these two values. Selecting galaxies based upon luminous infrared activity clearly biases the sample towards merging galaxies with small physical separations. However, the existence of pairs with large separations indicates that the ultraluminous phase may in some cases start early during the merger process. Alternatively, these systems may contain unresolved third nuclei responsible for triggering the ultraluminous activity. We briefly compare our results to recent models of merging spiral galaxies. (c) 1996 American Astronomical Society.
\end{abstract}

\section{INTRODUCTION}

The IRAS all sky survey revealed a class of galaxies with far-infrared luminosities comparable to the minimum accepted bolometric luminosities of quasars. These galaxies, which typically emit between $50 \%$ and $99 \%$ of their energy in the far-infrared, have $L_{\mathrm{IR}} \geq 10^{12} L_{\odot}$, and are often referred to as "ultraluminous" infrared galaxies (ULIRGs). There are 10 ULIRGs in the IRAS Bright Galaxy Sample (hereafter referred to as BGS) of Soifer et al. $(1987 ; 1989)$, complete to $5.4 \mathrm{Jy}$ at $60 \mu \mathrm{m}$. The visual and infrared properties of this class of galaxies have been discussed by Armus et al. 1987, 1990, 1994; Sanders et al. 1988a, 1988b; Carico et al. 1990;
Majewski et al. 1993; and Lawrence et al. 1989, among others. Since nearly all of these ULIRGs have distorted visual morphologies, it has been suggested that galactic interactions are the trigger for the ultraluminous activity (Soifer et al. 1984; Sanders et al. 1988a). Indeed, $N$-body models of spiral-spiral encounters (e.g., Mihos \& Hernquist 1994, and references therein) indicate that large masses of molecular gas can build up in the central few kpc of an interacting system during the final stages of a merger. This gas can fuel either a starburst or, in principle, a nonthermal active galactic nucleus (AGN). Sanders et al. (1988a) suggest that ULIRGs evolve into optically recognizable quasars once the large amount of nuclear material has dispersed, possibly through 
the action of starburst-induced winds (see Heckman et al. 1990, 1993 for reviews). Although the imaging data clearly indicate galactic interactions are at work in the ULIRGs, the dusty circumnuclear environments can often be probed only at infrared or radio wavelengths. Knowledge of the detailed nuclear structure at 1 to a few kpc (i.e., the detection of close double nuclei) is important in the derivation of the merger time scale, crucial for estimating the lifetime of the ultraluminous phase. The number of ULIRGs in a given volume of space along with an estimate of the time spent in the ultraluminous infrared state can place strong constraints on theories which posit an evolutionary link between these galaxies and quasars. Both visual and near-infrared imaging are therefore valuable for understanding the origin and ultimate fate of the ULIRGs.

Seven out of ten of the BGS ULIRGs have well separated double nuclei (Graham et al. 1990; Majewski et al. 1993; and Armus et al. 1994). Carico et al. (1990) used the measured separations of the ULIRGs with known double nuclei to estimate the lifetime of the ultraluminous phase. However, the large range in the physical nuclear separations in these double nucleus systems together with the small number of galaxies introduces a correspondingly large uncertainty in the estimate of the time until merger as well as the lifetime of the ultraluminous phase. The projected physical separations of these 7 galaxies range from $0.3 \mathrm{kpc}$ (Arp 220) to $5.8 \mathrm{kpc}$ (IRAS $08572+3915$ ), leading to more than an order of magnitude spread in the estimated merger time scales from $1.3 \times 10^{7} \mathrm{yr}$ for Arp 220 (Graham et al. 1990; Larkin et al. 1995 ) to $3.2 \times 10^{8} \mathrm{yr}$ for IRAS $08572+3915$. Infrared observations of Mrk 231 (Armus et al. 1994) further suggest that the two nuclei in this system may not merge for up to $10^{9} \mathrm{yr}$, although the dynamics of this unique system are highly uncertain.

To better determine the fraction of interacting and merging ULIRGs, the typical merger time scale, and the lifetime of the ultraluminous phase, we have undertaken a program to image a much larger sample of ULIRGs from the complete sample of Strauss et al. $(1990,1992)$. We have imaged 46 ULIRGs in the Gunn $r$ band using the Palomar 60 in. telescope. In addition, we have imaged 45 of the same galaxies in the near-infrared $K$ band using the Palomar 200 in. Hale telescope. The results of this imaging program are presented here. The major goals of this paper are to (1) estimate the fraction of sample galaxies with evidence for past or current interactions and (2) determine the lifetime of the ultraluminous infrared phase for the sample as a whole. In Sec. 2 we define the sample and describe the observations and production of the final images, and in Sec. 3 we present the visual and infrared data along with brief comments on the morphologies and visual luminosities of the ULIRGs as a class. In Sec. 4 we present a basic model for the evolution of a ULIRG, and use this to estimate both an upper and lower limit to the lifetime of the ultraluminous infrared phase. We compare these lifetimes to other time scale estimates based on the rate at which fuel is consumed to power the infrared emission. Finally, in Sec. 5 we summarize our results and conclusions. More detailed analysis of the infrared images as well as visual and infrared spectroscopic data for these galaxies will be presented in forthcoming papers in this series.

\section{SAMPLE \& OBSERVATIONS}

\subsection{The Northern Hemisphere 2 Jy ULIRG Sample}

We have chosen a sample of galaxies from the complete 2 Jy IRAS flux-limited sample of Strauss et al. (1990, 1992). This sample includes, as a subset, the bright ULIRGs found in the BGS. The 2 Jy sample contains 87 galaxies meeting the following criteria:

(1) $60 \mu \mathrm{m}$ flux density greater than $1.94 \mathrm{Jy}$.

(2) Flux quality at $60 \mu \mathrm{m}$ equal to 2 or 3 (moderate or high as defined in the IRAS Point Source Catalog, Explanatory Supplement).

(3) $F_{\nu}^{2}(60 \mu \mathrm{m})>F_{\nu}(12 \mu \mathrm{m}) \times F_{\nu}(25 \mu \mathrm{m})$, where $F_{\nu}(\lambda)$ is the IRAS Point Source Catalog flux density.

(4) Far-infrared luminosity $L_{\mathrm{FIR}}$ greater than $5.0 \times 10^{11}$ $L_{\odot}$.

(5) Absolute Galactic latitude, $|b|>5^{\circ}$.

The far-infrared luminosity is taken to be $L_{\mathrm{FIR}} \equiv 3.86 \times 10^{5} d^{2}\left[2.58 F_{\nu}(60 \mu \mathrm{m})+F_{\nu}(100 \mu \mathrm{m})\right] L_{\odot}$, where $d$ is the luminosity distance in Mpc, and $F_{\nu}(\lambda)$ is the IRAS flux density in Janskys. Throughout this paper, we use $H_{0}=75 \mathrm{~km} \mathrm{~s}^{-1} \mathrm{Mpc}^{-1}$ and $q_{0}=0$. We have chosen a farinfrared luminosity selection criterion of $L_{\mathrm{FIR}}>5.0 \times 10^{11} L_{\odot}$ to ensure a minimum bolometric luminosity of roughly $10^{12}$ $L_{\odot}$. This measure has the advantage of using the two IRAS bands $(60$ and $100 \mu \mathrm{m})$ where all the sample galaxies have confident detections. For the ULIRGs, the bolometric luminosity is typically a factor of 2 greater than $L_{\mathrm{FIR}}$.

Of the 87 ULIRGs meeting the above criteria, 64 have declinations $\delta \geqslant-35^{\circ}$, and are thus accessible to telescopes in the northern hemisphere. Ten of these galaxies are the well-studied members of the BGS (Sanders et al. 1988a), while seven (three of which are also in the BGS) are members of the Warm Galaxy Sample of Sanders et al. (1988b). It should be mentioned that although the well studied quasar 3C 273 meets all of the selection criteria, we have excluded it from the sample as it is clearly a quasar rather than an ultraluminous galaxy. For clarity, we will refer to the $542 \mathrm{Jy}$ ULIRGs which are not in the BGS as the faint ULIRG sample, and the total set of 64 galaxies as the northern hemisphere 2 Jy ULIRG sample. Basic data on these 64 galaxies, including heliocentric velocities, IRAS flux densities, and infrared luminosities (see below) are presented in Table 1 . The heliocentric velocities of the BGS galaxies are taken directly from Sanders et al. (1988a), except in the cases of IRAS $08572+3915$, IRAS 09320+6134, and IRAS 15250+3609, for which the heliocentric velocities (as for all the remaining faint sample galaxies) are taken from Strauss et al. (1992). All far-infrared flux densities are taken from the IRAS Point Source Catalog except in the case of the BGS and Warm Galaxy Sample galaxies for which the more accurate coadded flux densities have been taken directly from Sanders et al. (1988a, 1988b).

In Table 1 and throughout the remainder of this paper, we refer to the infrared luminosity, $L_{\mathrm{IR}}$, which is equivalent to the luminosity from 8 to $1000 \mu \mathrm{m}$, and is defined as 
TABLE 1. 2 Jy ULIRGs-Fluxes and luminosities.

\begin{tabular}{|c|c|c|c|c|c|c|}
\hline \multirow[b]{2}{*}{ Object Name } & \multirow[b]{2}{*}{$\mathrm{cz}(\mathrm{km} / \mathrm{s})$} & \multirow[b]{2}{*}{$\log \left(\mathrm{L}_{\mathrm{IR}} / \mathrm{L}_{\odot}\right)$} & \multicolumn{4}{|c|}{$\mathrm{F}_{\nu}(\lambda)[\mathrm{Jy}]$} \\
\hline & & & $12 \mu \mathrm{m}$ & $25 \mu \mathrm{m}$ & $60 \mu \mathrm{m}$ & $100 \mu \mathrm{m}$ \\
\hline IRAS $00091-0738$ & 35509 & 12.21 & $0.25 \mathrm{~L}$ & $0.36 \mathrm{~L}$ & 2.75 & 2.50 \\
\hline IRAS $00153+5454$ & 33448 & 12.10 & $0.25 \mathrm{~L}$ & $0.26 \mathrm{~L}$ & 2.22 & 2.72 \\
\hline IRAS $00188-0856$ & 38550 & 12.33 & $0.25 \mathrm{~L}$ & $0.66 \mathrm{~L}$ & 2.85 & 3.35 \\
\hline IRAS $00262+4251$ & 29129 & 12.08 & $0.25 \mathrm{~L}$ & 0.34 & 3.03 & 2.57 \\
\hline IRAS 00456-2904 & 33060 & 12.16 & $0.25 \mathrm{~L}$ & $0.25 \mathrm{~L}$ & 2.55 & 3.48 \\
\hline IRAS $01003-2238 \dagger$ & 35286 & 12.25 & 0.19 & 0.56 & 2.24 & 1.79 \\
\hline IRAS 01298-0744 & 40826 & 12.29 & $0.39 \mathrm{~L}$ & $0.45 \mathrm{~L}$ & 2.50 & 2.23 \\
\hline IRAS $01521+5224$ & 23957 & 11.95 & $0.25 \mathrm{~L}$ & $0.25 \mathrm{~L}$ & 3.01 & 4.37 \\
\hline IRAS $01572+0009 \dagger$ & 48869 & 12.55 & 0.11 & 0.63 & 2.34 & 2.29 \\
\hline IRAS $03158+4227$ & 40288 & 12.55 & $0.25 \mathrm{~L}$ & 0.48 & 4.39 & 4.62 \\
\hline IRAS $03521+0028$ & 45622 & 12.48 & $0.25 \mathrm{~L}$ & 0.23 & 2.64 & 3.83 \\
\hline IRAS $04232+1436$ & 23972 & 11.99 & $0.25 \mathrm{~L}$ & $0.38 \mathrm{~L}$ & 3.45 & 4.26 \\
\hline IRAS $05189-2524 * \dagger$ & 12816 & 12.11 & 0.76 & 3.52 & 13.94 & 11.68 \\
\hline IRAS $05246+0103$ & 29037 & 12.05 & $0.25 \mathrm{~L}$ & 0.56 & 2.56 & 1.96 \\
\hline IRAS $08030+5243$ & 25006 & 11.97 & $0.25 \mathrm{~L}$ & 0.22 & 3.00 & 4.24 \\
\hline IRAS $08311-2459$ & 29988 & 12.40 & $0.33 \mathrm{~L}$ & 1.10 & 4.96 & 5.80 \\
\hline IRAS $08344+5105$ & 29028 & 11.94 & $0.25 \mathrm{~L}$ & $0.25 \mathrm{~L}$ & 2.06 & 2.60 \\
\hline IRAS $08572+3915 * \dagger$ & 17480 & 12.08 & 0.35 & 1.73 & 7.53 & 4.59 \\
\hline IRAS 09061-1248 & 22073 & 11.97 & $0.25 \mathrm{~L}$ & $0.26 \mathrm{~L}$ & 3.67 & 5.50 \\
\hline IRAS 09111-1007 & 16438 & 11.98 & $0.25 \mathrm{~L}$ & 0.47 & 7.47 & 10.46 \\
\hline IRAS $09320+6134 *$ & 12000 & 11.95 & 0.25 & 1.05 & 12.09 & 20.07 \\
\hline IRAS $09583+4714$ & 25717 & 11.98 & $0.25 \mathrm{~L}$ & 0.51 & 2.78 & 2.96 \\
\hline IRAS $10035+4852$ & 19371 & 11.93 & $0.25 \mathrm{~L}$ & 0.32 & 4.78 & 6.27 \\
\hline IRAS $10190+1322$ & 22987 & 11.98 & $0.25 \mathrm{~L}$ & $0.54 \mathrm{~L}$ & 3.33 & 5.39 \\
\hline IRAS $10378+1109$ & 40843 & 12.23 & $0.25 \mathrm{~L}$ & $0.34 \mathrm{~L}$ & 2.21 & 1.84 \\
\hline IRAS $10494+4424$ & 27616 & 12.15 & $0.25 \mathrm{~L}$ & $0.25 \mathrm{~L}$ & 3.36 & 5.60 \\
\hline IRAS $10565+2448$ & 12926 & 11.98 & $0.28 \mathrm{~L}$ & 1.16 & 12.17 & 14.34 \\
\hline IRAS $11095-0238$ & 31796 & 12.20 & $0.25 \mathrm{~L}$ & 0.46 & 3.23 & 2.76 \\
\hline IRAS $11506+1331$ & 38206 & 12.27 & $0.25 \mathrm{~L}$ & $0.56 \mathrm{~L}$ & 2.46 & 3.14 \\
\hline IRAS 11598-0112 & 45177 & 12.40 & $0.36 \mathrm{~L}$ & $0.54 \mathrm{~L}$ & 2.41 & 2.73 \\
\hline IRAS 12071-0444† & 38480 & 12.31 & $0.14 \mathrm{~L}$ & 0.53 & 2.57 & 2.19 \\
\hline IRAS $12112+0305 *$ & 21788 & 12.27 & $0.18 \mathrm{~L}$ & 0.63 & 8.36 & 9.91 \\
\hline IRAS $12540+5708 * \dagger$ & 12900 & 12.50 & 1.81 & 8.52 & 33.60 & 30.89 \\
\hline IRAS $13428+5608 *$ & 11132 & 12.10 & 0.31 & 2.32 & 23.70 & 22.31 \\
\hline IRAS $13451+1232 \dagger$ & 36341 & 12.27 & 0.16 & 0.66 & 2.01 & 2.14 \\
\hline IRAS $13539+2920$ & 32513 & 12.04 & $0.25 \mathrm{~L}$ & $0.25 \mathrm{~L}$ & 1.97 & 2.69 \\
\hline IRAS $14348-1447 *$ & 24732 & 12.28 & 0.09 & 0.56 & 6.82 & 7.49 \\
\hline IRAS 14352-1954 & 26886 & 11.95 & $0.25 \mathrm{~L}$ & $0.31 \mathrm{~L}$ & 2.31 & 3.42 \\
\hline IRAS $14394+5332$ & 31450 & 12.04 & $0.25 \mathrm{~L}$ & 0.33 & 2.08 & 2.36 \\
\hline IRAS $15001+1433$ & 48790 & 12.38 & $0.25 \mathrm{~L}$ & $0.25 \mathrm{~L}$ & 1.98 & 2.16 \\
\hline IRAS $15130-1958$ & 32792 & 12.03 & $0.25 \mathrm{~L}$ & $0.40 \mathrm{~L}$ & 1.96 & 2.43 \\
\hline IRAS $15245+1019$ & 22687 & 11.96 & $0.25 \mathrm{~L}$ & 0.27 & 3.54 & 5.21 \\
\hline IRAS $15250+3609 *$ & 16602 & 11.99 & 0.15 & 1.32 & 7.50 & 5.86 \\
\hline IRAS $15327+2340 *$ & 5450 & 12.11 & 0.46 & 8.11 & 104.08 & 117.69 \\
\hline IRAS 15462-0405 & 30150 & 12.16 & $0.25 \mathrm{~L}$ & 0.48 & 3.23 & 3.03 \\
\hline IRAS 16090-0139 & 40029 & 12.48 & $0.25 \mathrm{~L}$ & 0.27 & 3.72 & 4.72 \\
\hline IRAS $16474+3430$ & 33418 & 12.12 & $0.25 \mathrm{~L}$ & 0.25 & 2.27 & 2.89 \\
\hline IRAS $16487+5447$ & 31293 & 12.12 & $0.25 \mathrm{~L}$ & 0.18 & 2.87 & 3.30 \\
\hline IRAS $17028+5817$ & 31779 & 12.11 & $0.25 \mathrm{~L}$ & 0.08 & 2.49 & 4.05 \\
\hline IRAS 17208-0014 & 12836 & 12.38 & $0.25 \mathrm{~L}$ & 1.67 & 34.09 & 35.71 \\
\hline IRAS $17574+0629$ & 32860 & 12.10 & $0.25 \mathrm{~L}$ & 0.38 & 2.21 & 2.35 \\
\hline IRAS $18368+3549$ & 34825 & 12.19 & $0.25 \mathrm{~L}$ & $0.25 \mathrm{~L}$ & 2.23 & 3.83 \\
\hline IRAS $18443+7433$ & 40395 & 12.23 & $0.25 \mathrm{~L}$ & 0.17 & 2.15 & 2.24 \\
\hline IRAS $18470+3233$ & 23626 & 12.02 & $0.25 \mathrm{~L}$ & 0.44 & 4.25 & 3.43 \\
\hline IRAS 19297-0406 & 25674 & 12.36 & $0.25 \mathrm{~L}$ & 0.65 & 7.33 & 8.33 \\
\hline IRAS $19458+0944$ & 29848 & 12.31 & $0.25 \mathrm{~L}$ & $0.28 \mathrm{~L}$ & 3.95 & 7.11 \\
\hline IRAS 20046-0623 & 25362 & 12.02 & $0.25 \mathrm{~L}$ & $0.50 \mathrm{~L}$ & 3.34 & 4.06 \\
\hline IRAS $20087-0308$ & 31600 & 12.39 & $0.25 \mathrm{~L}$ & $0.27 \mathrm{~L}$ & 4.61 & 6.53 \\
\hline IRAS $20414-1651$ & 26107 & 12.19 & $0.25 \mathrm{~L}$ & $0.41 \mathrm{~L}$ & 4.69 & 5.28 \\
\hline IRAS $21396+3623$ & 29034 & 12.00 & $0.25 \mathrm{~L}$ & $0.25 \mathrm{~L}$ & 2.16 & 3.52 \\
\hline IRAS 21504-0628 & 23263 & 11.94 & $0.25 \mathrm{~L}$ & $0.51 \mathrm{~L}$ & 3.70 & 3.04 \\
\hline IRAS $22491-1808 *$ & 23170 & 12.12 & $0.12 \mathrm{~L}$ & 0.57 & 5.54 & 4.64 \\
\hline IRAS $23327+2913$ & 31981 & 12.03 & $0.25 \mathrm{~L}$ & $0.35 \mathrm{~L}$ & 2.08 & 2.59 \\
\hline IRAS $23365+3604$ & 19338 & 12.13 & $0.25 \mathrm{~L}$ & 0.81 & 7.69 & 8.19 \\
\hline
\end{tabular}

* Members of the Bright Galaxy Sample (BGS) studied by Sanders et al. (1988a).

Col. 1: IRAS designation.

$\dagger$ Members of the Warm Galaxy Sample (Sanders et al., 1988b).

Col. 2: Heliocentric recessional velocity, in $\mathrm{km} \mathrm{s}^{-1}$, taken from Strauss et al. (1992), except for seven of the ten BGS members (marked by asterisks), which come from Sanders et al. (1988a) (see text for details).

Col. 3: Logarithm of the infrared luminosity $L_{\mathrm{IR}}$ as defined in the text. When only limits are available for the IRAS flux densities (typically 12 and/or $25 \mu \mathrm{m}$ ), $L_{\mathrm{IR}}$ is estimated based on median flux ratios of bright ULIRGs with IRAS flux detections in all four bands. In all cases, we have used $H_{0}=75 \mathrm{~km} \mathrm{~s}^{-1} \mathrm{Mpc}^{-1}$ and $q_{0}=0$.

Cols. 4-7: IRAS flux densities, in Janskys, from the IRAS Point Source Catalog. The flux densities for the BGS ULIRGs are from Sanders et al. (1988a), and those for the members of the Warm Galaxy Sample are from Sanders et al. (1988b). Numbers with an "L" appended represent upper limits of the flux density. 


$$
\begin{aligned}
L_{\mathrm{IR}}= & 5.5 \times 10^{5} d^{2}\left[13.48 F_{\nu}(12 \mu \mathrm{m})+5.16 F_{\nu}(25 \mu \mathrm{m})\right. \\
& \left.+2.58 F_{\nu}(60 \mu \mathrm{m})+F_{\nu}(100 \mu \mathrm{m})\right] L_{\odot}
\end{aligned}
$$

(IRAS Point Source Catalog, Explanatory Supplement, p. $\mathrm{X}-13)$ with $d$ and $F_{\nu}(\lambda)$ defined as above. Although we have used only the 60 and $100 \mu \mathrm{m}$ IRAS flux densities to select the galaxies based upon $L_{\mathrm{FIR}}$, inclusion of the 12 and $25 \mu \mathrm{m}$ bands provides a better estimate of the total infrared (and hence bolometric) luminosity of the ULIRGs. When the IRAS $12 \mu \mathrm{m}$ flux density is given as an upper limit, we assume that the missing flux contributes $7 \%$ to the total infrared luminosity. When both the 12 and $25 \mu \mathrm{m}$ flux densities are upper limits, we assume that the combined flux from these two bands contributes $19 \%$ to the infrared luminosity. These fractional flux contributions were determined from the energy distributions of the eleven brightest ULIRGs with confident detections in all four IRAS bands.

\subsection{Observations \& Data Reduction}

Near-infrared images of 45 of the 54 sample galaxies were taken at the $f / 70$ focus of the Hale 200 in. telescope between 1993 July and 1994 February using a $K$ band filter $(2.23 \mu \mathrm{m}$ central wavelength, FWHM $\approx 0.40 \mu \mathrm{m})$. Two cameras were employed to obtain these images, one which uses a $58 \times 62 \mathrm{InSb}$ array manufactured by Santa Barbara Research Corporation and one which uses a $256 \times 256 \mathrm{HgCdTe}$ array manufactured by Rockwell Science Center. The projected scale of the InSb array is 0.313 pixel $^{-1}$, while that of the $\mathrm{HgCdTe}$ array is 0.167 pixel $^{-1}$. Sky conditions were generally not photometric during the acquisition of these images. For each galaxy, observations of a nearby star were made in order to obtain an accurate estimate of the point spread function (PSF) at the time of observation. The seeing, measured from the PSF, was typically $\sim 0$ " 8 , and is presented in Table 2 for each galaxy. Integration times per frame ranged between 20 and $40 \mathrm{~s}$. Each frame underwent linearization, bias subtraction, flat-field correction, and sky subtraction. Images of the twilight sky were used to generate the flat-field frames. Multiple exposures of each galaxy (typically five) were aligned and then coadded by either centroiding on the galaxy nucleus (when bright in individual frames) or using the relative positions of the offset guider. The 45 final, coadded $K$ band images, each typically covering $12.8^{\prime \prime} \times 12.8^{\prime \prime}$, are displayed in Fig. 1.

Visual images were obtained at the $f / 8.75$ Cassegrain focus of the Palomar 60 in. telescope. All images were taken with a Gunn $r$ filter (6550 ^ central wavelength, FWHM $\approx 900 \AA$ A Thuan \& Gunn 1976) using two Tektronics CCDs $(1024 \times 1024$ and $2048 \times 2048)$ both with a scale of 0.376 pixel $^{-1}$. All images were taken between 1993 February and 1994 July. Integrations were typically $600 \mathrm{~s}$ long. Nearly all of the $r$ band images were taken under photometric conditions. These images were bias subtracted, flat fielded and sky subtracted in the usual manner. The normalized flat-field frames were produced from exposures of the illuminated dome. After these reduction steps, the individual galaxy exposures (typically 2-3 per galaxy) were aligned and coadded by centroiding on stars in the field. The final $46 r$ band images, each typically covering $30^{\prime \prime} \times 30^{\prime \prime}$, are presented in Fig. 1. In some cases individual $r$ band images cover a larger portion of the sky to accommodate very extended systems.

\section{RESULTS \\ 3.1 Morphologies}

In order to better understand the role of the interaction and merger on the generation of ultraluminous infrared activity, we have attempted to place each of the sample galaxies into a simple morphological category. The categories are: single ("S"), double ("D"), multiple ("M"), or wide pair ("W"). The wide pair classification ("W") represents pairs of galaxies showing no obvious evidence for interaction in the $r$ band images. Some single ("S") and wide pair ("W") galaxies have an additional designation of " $t$ " if the system appears to exhibit tidal tails, or " $u$ " if the system appears undistorted. Nuclear separations of all the sample galaxies with apparent double nuclei have been measured. Table 2 presents a summary of these classifications for the galaxies imaged in this paper, along with nuclear separations derived from the images. In Table 3 the same information is presented for the ten BGS ULIRGs. Here, the infrared morphologies are taken from Carico et al. (1990), Graham et al. (1990), Majewski et al. (1993), and Armus et al. (1994). In Appendix A we discuss in more detail the motivation behind the classification of several of the more morphologically complex targets.

It is obvious from Fig. 1 that the vast majority (43/46, or 93\%) of the faint sample ULIRGs for which we have obtained images has distorted morphologies, unlike the majority of elliptical or spiral galaxies. Including the ten BGS ULIRGs, a total of 53 out of $56(95 \%)$ of the systems show evidence for very distorted morphologies. Among the interacting systems, there are clear cases of luminous arcs or bridges connecting widely separated pairs, long stellar tails, and irregular inner isophotes with multiple, bright peaks. Since the relation between enhanced far-infrared emission and galactic interactions is now well established (see, for example, Joseph \& Wright 1984; Lonsdale et al. 1984; Soifer et al. 1984; Cutri \& McAlary 1985; Armus et al. 1987; and Sanders et al. 1988a), it is not surprising that the majority of the 2 Jy ULIRGs consists of galaxies apparently in the throes of very disruptive galaxy-galaxy collisions.

The three galaxies which do not appear disturbed (classified with a "u" in Table 2) are IRAS 01003-2238, IRAS $03158+4227$, and IRAS $08030+5243$. The latter two galaxies have bright, nearby stars evident in Fig. 1 which may be hiding faint distorted outer isophotes or other tidal features. IRAS 01003-2238, has two nearby, resolved, apparently galactic companions at radii of about $15^{\prime \prime}$, but no obvious signs of either current or past interactions.

A large number of the 2 Jy ULIRGs appear to be double nucleus systems. Of the 56 sample galaxies with imaging data, $25(45 \%)$ have resolved double nuclei. In order to be considered a double nucleus system, both nuclei must be visible and spatially resolved in the $K$ band image, except for the cases (e.g., IRAS 14394+5332, IRAS 23327+2913) where the nuclear separation is larger than the $K$ band field. 
TABLE 2. 2 Jy ULIRGs-Infrared and optical imaging.

\begin{tabular}{|c|c|c|c|c|c|c|c|}
\hline Object Name & $\begin{array}{c}\text { Infrared } \\
\text { Morphology }\end{array}$ & $\begin{array}{c}\text { Optical } \\
\text { Morphology }\end{array}$ & sep. $\left({ }^{\prime \prime}\right)$ & sep. $(\mathrm{kpc})$ & $\mathrm{m}_{\mathrm{r}}$ & $\overline{M_{r}}$ & $\begin{array}{c}\text { Notes on } \\
\text { magnitudes }\end{array}$ \\
\hline IRAS $00091-0738$ & $\mathrm{D}$ & $\mathrm{S}$ & $1.2[0.7]$ & 2.3 & - & - & \\
\hline IRAS $00188-0856$ & $\mathrm{D}$ & $\mathrm{D}$ & $7.0[0.8]$ & 14.1 & 16.8 & -22.4 & 1 \\
\hline IRAS $00262+4251$ & $\mathrm{~S}$ & $\mathrm{~S}$ & $<0.8$ & $<1.3$ & 16.0 & -22.5 & \\
\hline IRAS $00456-2904$ & $\mathrm{~S}$ & St & $<1.1$ & $<2.0$ & - & - & \\
\hline IRAS $01572+0009$ & $\mathrm{~S}$ & St & $<0.8$ & $<2.0$ & 15.6 & -24.4 & \\
\hline IRAS $03158+4227$ & $\mathrm{~S}$ & Wu & $<0.8$ & $<1.6$ & 17.7 & -21.7 & 2 \\
\hline IRAS $03521+0028$ & $\mathrm{D}$ & $\mathrm{S}$ & $1.6[0.8]$ & 3.6 & 18.4 & -21.4 & \\
\hline IRAS $05246+0103$ & $\mathrm{D}$ & $\mathrm{D}$ & $6.0[0.7]$ & 9.6 & 15.9 & -22.6 & \\
\hline IRAS $08030+5243$ & $\mathrm{~S}$ & $\mathrm{Su}$ & $<0.8$ & $<1.1$ & 17.0 & -21.0 & \\
\hline IRAS $08311-2459$ & $\mathrm{~S}$ & St & $<0.8$ & $<1.3$ & 14.7 & -23.9 & \\
\hline IRAS $10565+2448$ & M & M & $25.8[0.7]$ & 20.2 & $15.0,16.5$ & $-21.8,-20.3$ & 5 \\
\hline IRAS $11095-0238$ & $\mathrm{~S}$ & St & $<0.8$ & $<1.4$ & - & - & \\
\hline IRAS $11506+1331$ & $\mathrm{~S}$ & $\mathrm{~S}$ & $<0.5$ & $<1.1$ & 17.1 & -22.2 & 3 \\
\hline IRAS $11598-0112$ & $S$ & $\mathrm{~S}$ & $<0.6$ & $<1.3$ & 16.6 & -23.1 & \\
\hline IRAS $12071-0444$ & $\mathrm{D}$ & St & $0.9[0.6]$ & 1.8 & 16.6 & -22.6 & \\
\hline IRAS $13451+1232$ & $\mathrm{D}$ & $\mathrm{D}$ & $2.0[0.8]$ & 3.9 & 15.5 & -23.6 & \\
\hline IRAS $13539+2920$ & $\mathrm{D}$ & $\mathrm{D}$ & $4.1[0.8]$ & 7.2 & 16.9 & -21.9 & 1 \\
\hline IRAS $14394+5332$ & $\mathrm{~S}(\mathrm{D})$ & $\mathrm{D}$ & (28) $[1.0]$ & $(48)$ & $16.5,17.2$ & $-22.2,-21.5$ & 6 \\
\hline IRAS $15001+1433$ & $\mathrm{~S}$ & W & $<0.8$ & $<1.9$ & 17.0 & -23.0 & 3 \\
\hline IRAS $15130-1958$ & $\mathrm{~S}$ & St & $<0.8$ & $<1.3$ & 17.1 & -21.7 & \\
\hline IRAS $15245+1019$ & $\mathrm{D}$ & St & $2.6[0.7]$ & 3.4 & 15.8 & -22.1 & \\
\hline IRAS $18443+7433$ & $\mathrm{~S}$ & St & $<0.8$ & $<1.7$ & 17.3 & -21.0 & \\
\hline IRAS $18470+3233$ & M & M & $7.0[0.8]$ & 9.4 & 16.0 & -21.9 & 1 \\
\hline IRAS 19297-0406 & - & $\mathbf{S}$ & $(<1.9)$ & $(<2.7)$ & 16.0 & -22.2 & \\
\hline IRAS $19458+0944$ & $\mathrm{~S}$ & $\mathrm{D}$ & $<0.8$ & $<1.4$ & 16.1 & -22.4 & \\
\hline IRAS 20046-0623 & $\mathrm{S}$ & St & $<0.9$ & $<1.3$ & 16.3 & -21.8 & \\
\hline IRAS $20087-0308$ & $\mathrm{~S}$ & St & $<1.0$ & $<1.7$ & 16.1 & -22.6 & \\
\hline IRAS 20414-1651 & $\mathrm{S}$ & $\mathrm{D}$ & $<0.9$ & $<1.4$ & 17.1 & -21.1 & 8 \\
\hline IRAS $21396+3623$ & D & $\mathrm{D}$ & $6.7[0.8]$ & 10.7 & 16.5 & -22.0 & 1 \\
\hline IRAS $23327+2913$ & $\mathrm{~S}(\mathrm{D})$ & $\mathrm{D}$ & $(12.5)[0.7]$ & $(21.7)$ & $17.5,17.2$ & $-21.2,-21.5$ & 9 \\
\hline IRAS $23365+3604$ & $\mathrm{~S}$ & St & $<0.9$ & $<1.1$ & 15.0 & -22.4 & \\
\hline
\end{tabular}

Col. 1: IRAS designation.

Col. 2: Morphological classification of the $K$ band images. An "S" indicates an apparent single nucleus, "D" a double nucleus system, and "M" an apparent multiple nucleus system. An "S(D)" is used for cases in which the $K$-band frame is too small to show the second galaxy of the pair, which is obvious in the much larger $r$ band frame.

Col. 3: Morphological classification of the $r$ band images. The designations are the same as that for col. 2, with additional "t", " $u$ ", and "W" classifications representing galaxies with apparent tidal tails, undistorted systems, and widely separated pairs, respectively. The "W" systems have two bright galaxies which are not obviously interacting.

Col. 4: Separation of interacting pairs, measured in arcseconds from the $K$ band images. For single systems, the $K$ band seeing is used as an upper limit to the angular separation in the system. For pairs with measured separations, the $K$ band seeing is given in brackets for reference. Values in parentheses are measured from the $r$ band images.

Col. 5: Projected separation of pairs in kiloparsecs, obtained from the values in col. 4.

Col. 6: Apparent $r$ band magnitudes (corrected for Galactic extinction) for galaxies imaged under photometric conditions. For IRAS 00153+5454, IRAS 08311-2459, and IRAS 19458+0944, $|b|<10^{\circ}$, and the Galactic line-of-sight extinction is taken to be $\mathrm{E}(B-V) \sim 0.25$ mag (see text).

Col. 7: Absolute $r$ band magnitudes, calculated from the values in col. 6 as described in the text.

Col. 8: Notes on $r$ band magnitudes. Calculation of $r$ band magnitude: (1) includes both components in common envelope; (2) includes only the component in the IRAS error ellipse (see text); (3) includes only galaxy in box designating $K$ band field; (4) includes only galaxies within ellipse; (5) first magnitude for SW component, second for NE component; (6) first magnitude for E component, second for W component; (7) first magnitude for W component, second for E component; (8) excludes object to south; (9) first magnitude for S component, second for $\mathrm{N}$ component.

In addition, one or more of the following criteria has to be satisfied: (1) the two nuclei are encompassed by a common isophotal envelope well above the sky level (e.g., IRAS $00153+5454$, IRAS $21396+3623)$; (2) there is an obvious stellar bridge connecting the two nuclei (e.g., IRAS 14394 +5332, IRAS $23327+2913$ ); or (3) both nuclei are known to be at the same redshift. This last criterion is currently applicable only in the case of IRAS $17028+5817$, where two 

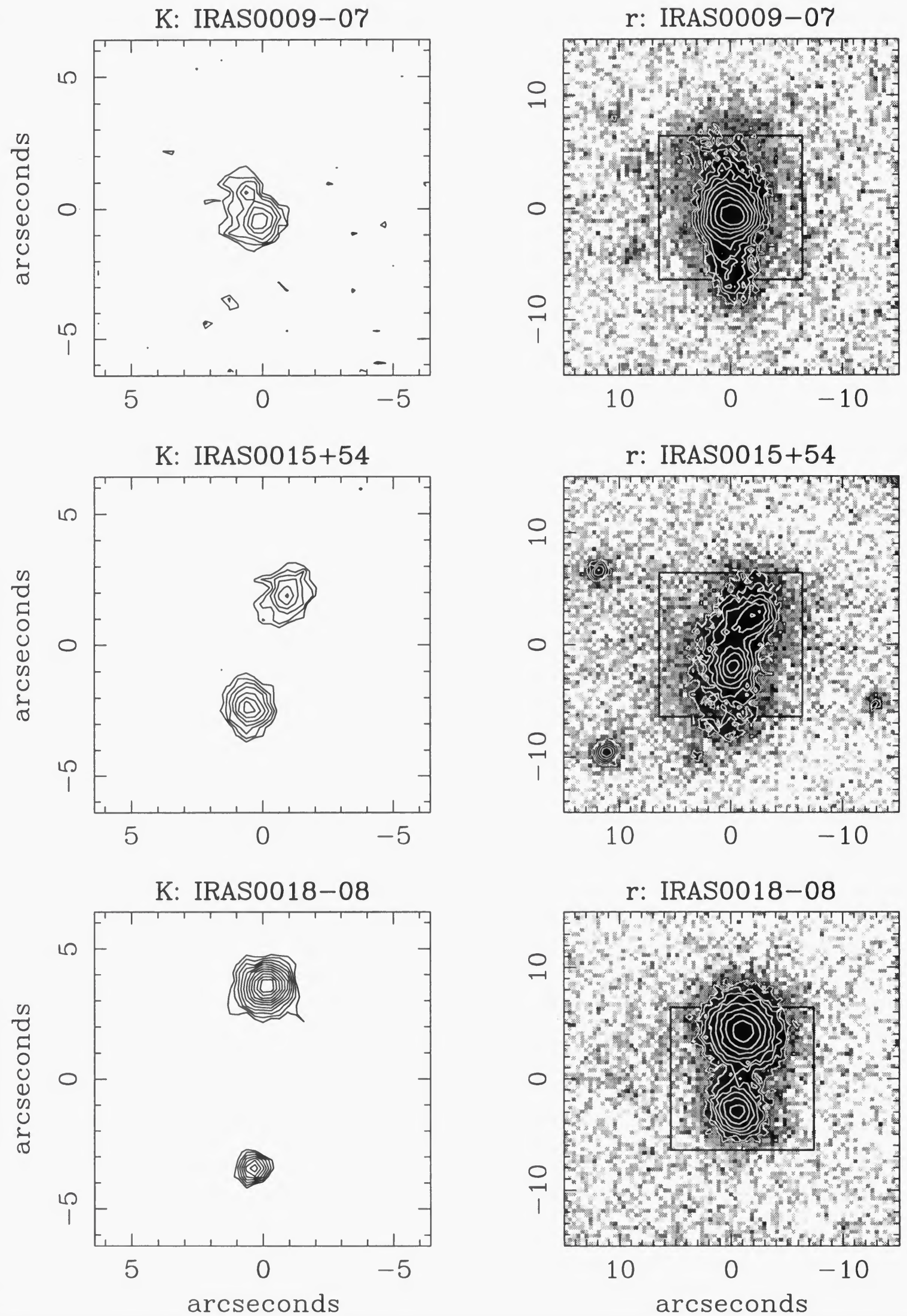

FIG. 1. Near-infrared and visual images for the $462 \mathrm{Jy}$ northern hemisphere ULIRGs imaged in this paper. The $K$ band images are represented as contour maps, with the lowest contour at the $3.0 \sigma$ level, and contour intervals separated by factors of 1.33 . The $K$ band frames are generally $12.8^{\prime \prime} \times 12.8^{\prime \prime}$, but vary slightly in size according to particular morphologies. The $r$ band images are plotted in both grayscale and contour form. The contours begin at the 3.0 $\sigma$ level (except for IRAS 09111-1007, IRAS 10565 +2448 , IRAS 17208-0014, IRAS $18368+3549$, IRAS $19297-0406$, and IRAS $19458+0944$, whose contours start at the $6 \sigma$ level), with contour intervals of factors of $\sqrt{2}$. Angular scale on the sky is indicated in arcsec, and a box appears in the $r$ band frame to show the size and location of the $K$ band frame. The $r$ band frames are typically $30^{\prime \prime} \times 30^{\prime \prime}$, but vary to accommodate different morphologies. IRAS positional uncertainty ellipses, at the $95 \%$ confidence level, have been drawn in $r$ band frames in which two or more bright galaxies appear. In all frames north is up and east is to the left. 

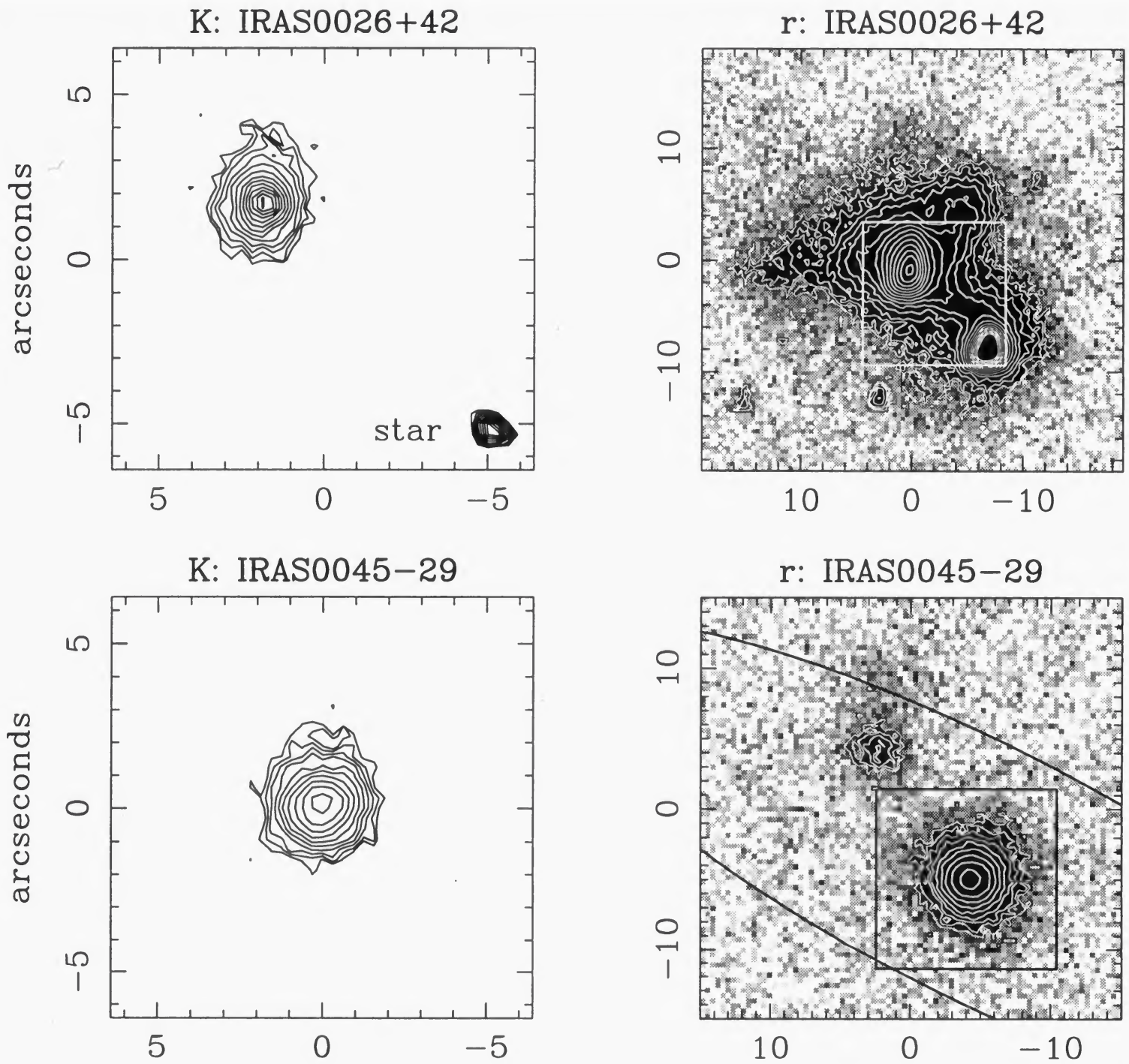

K: IRAS0100-22
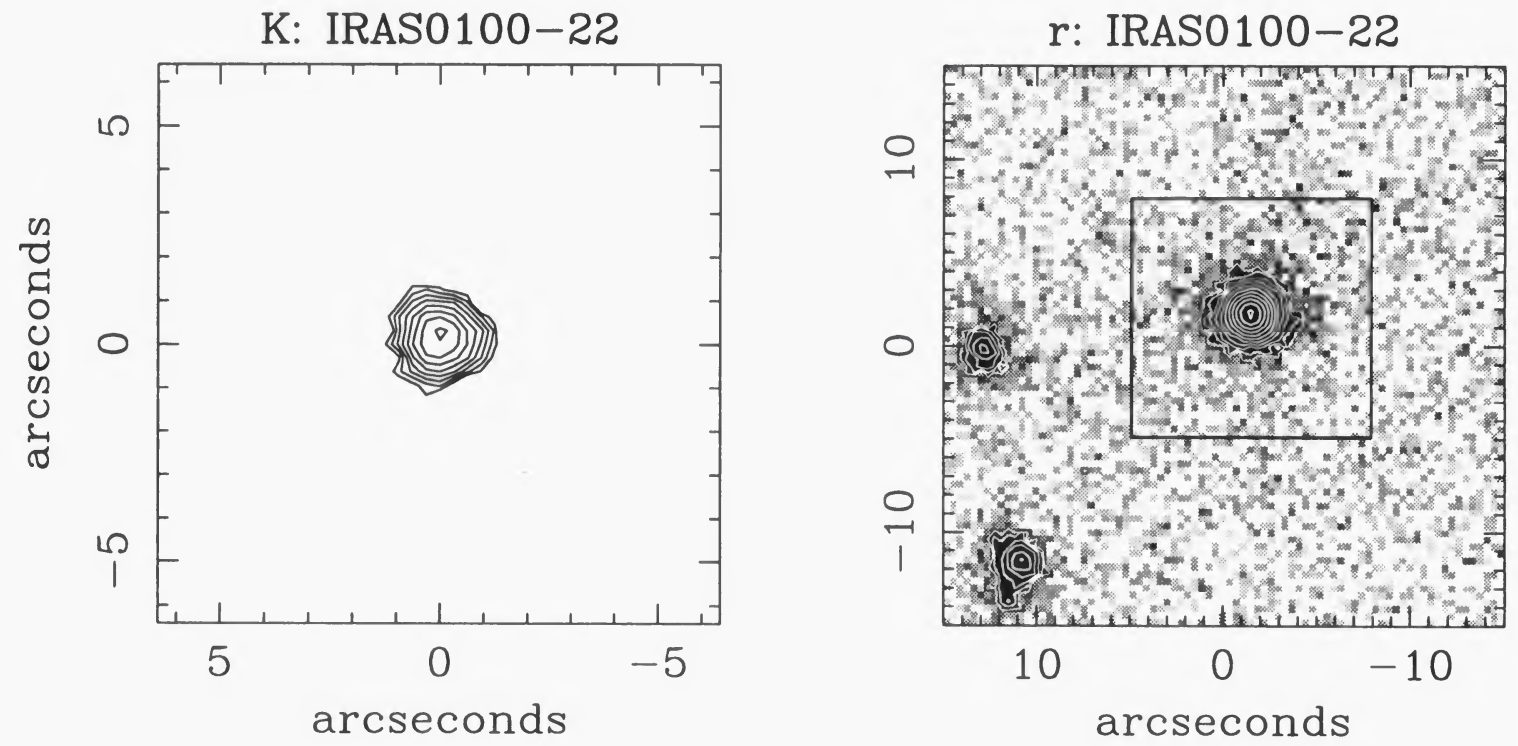

FIG. 1. (continued) 

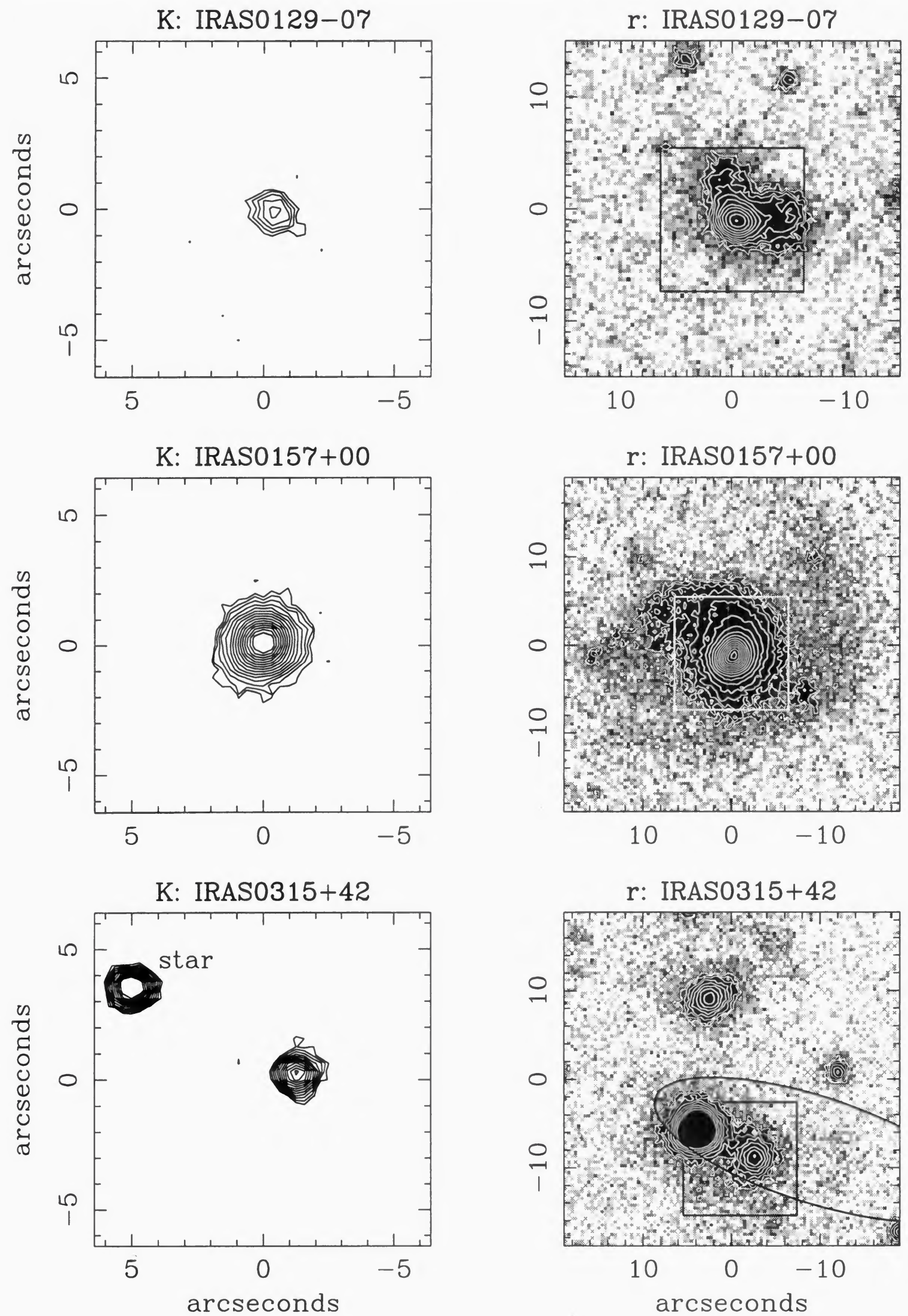

FIG. 1. (continued) 

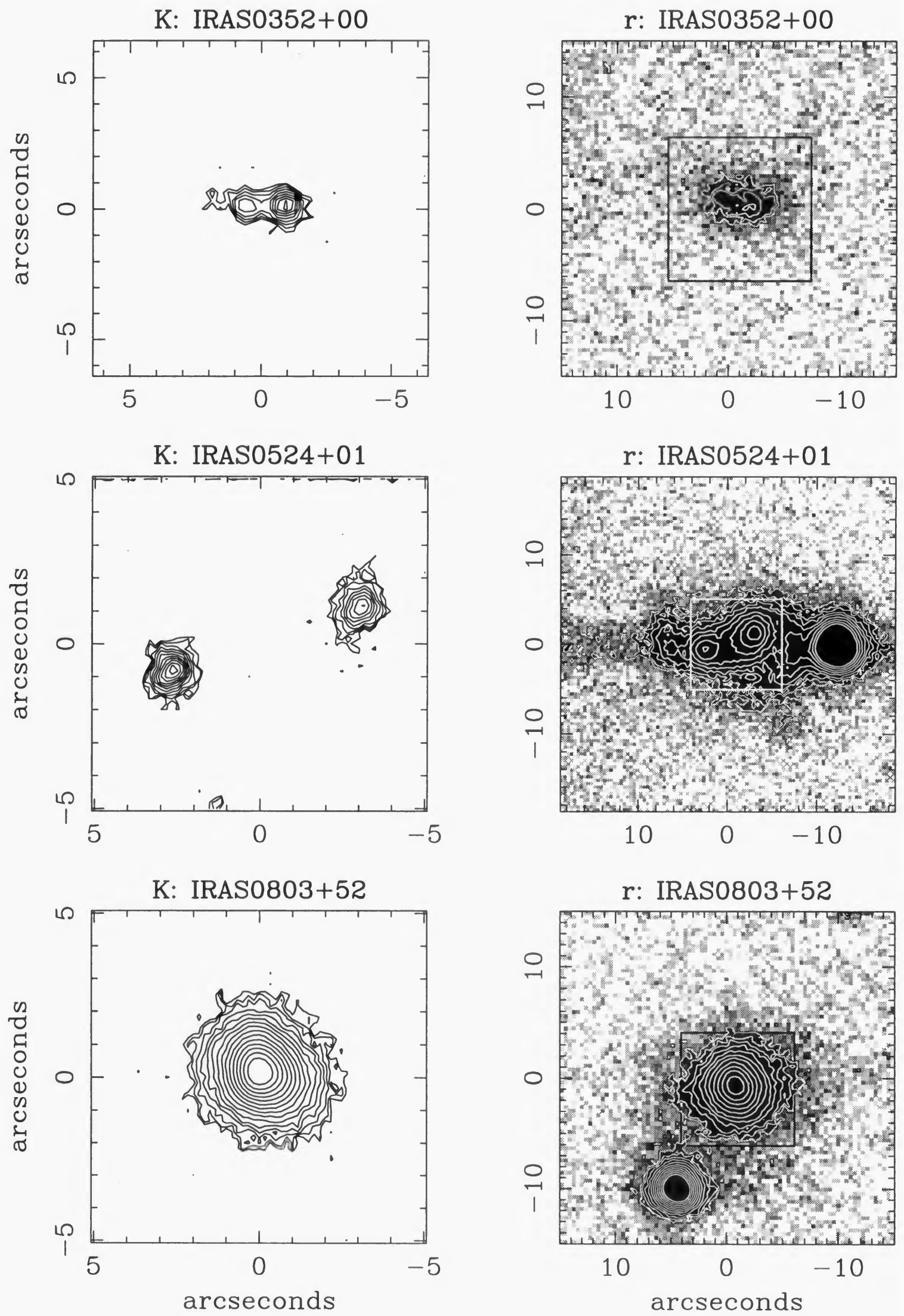

FIG. 1. (continued) 

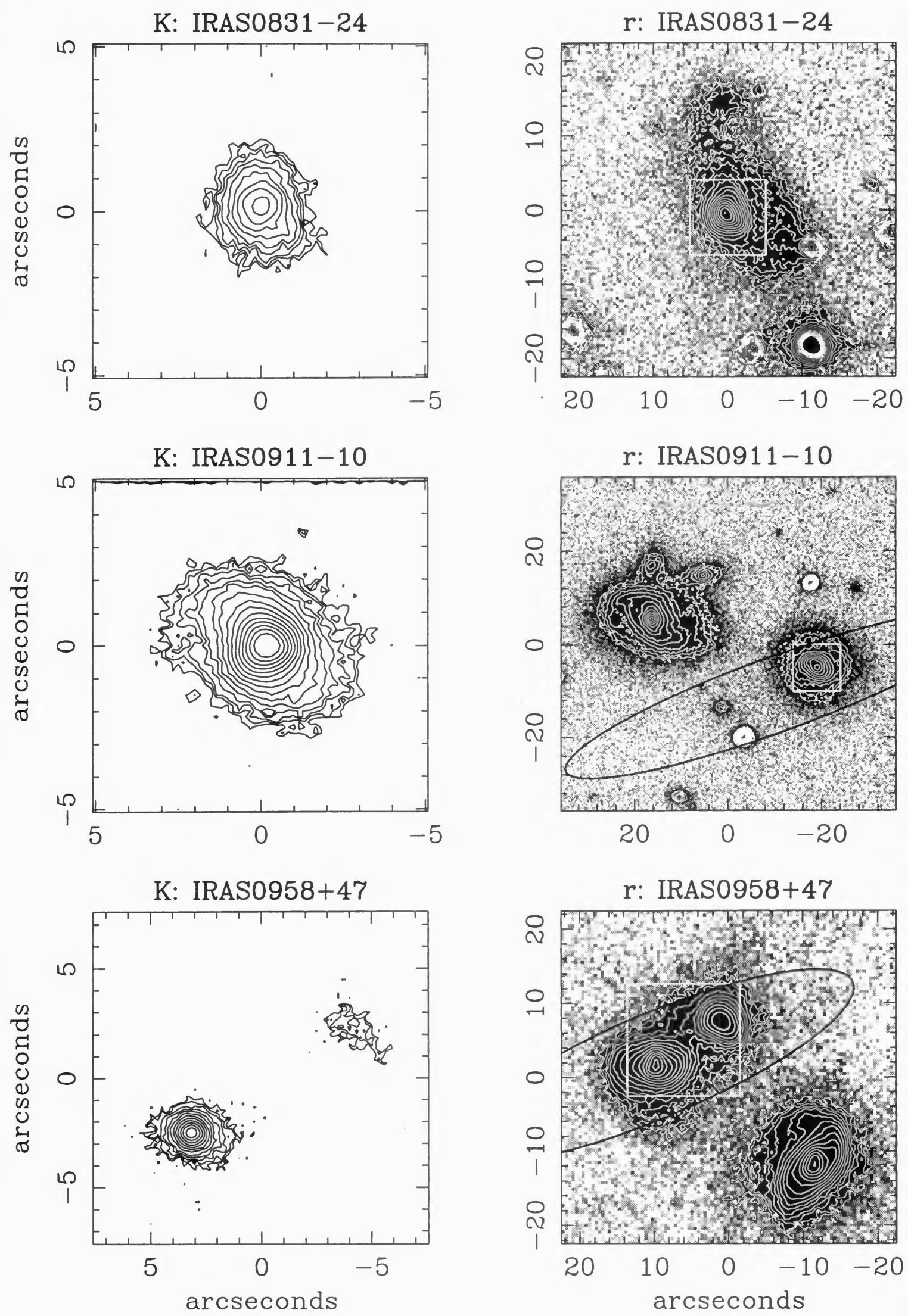

FIG. 1. (continued) 
K: IRAS1037+11

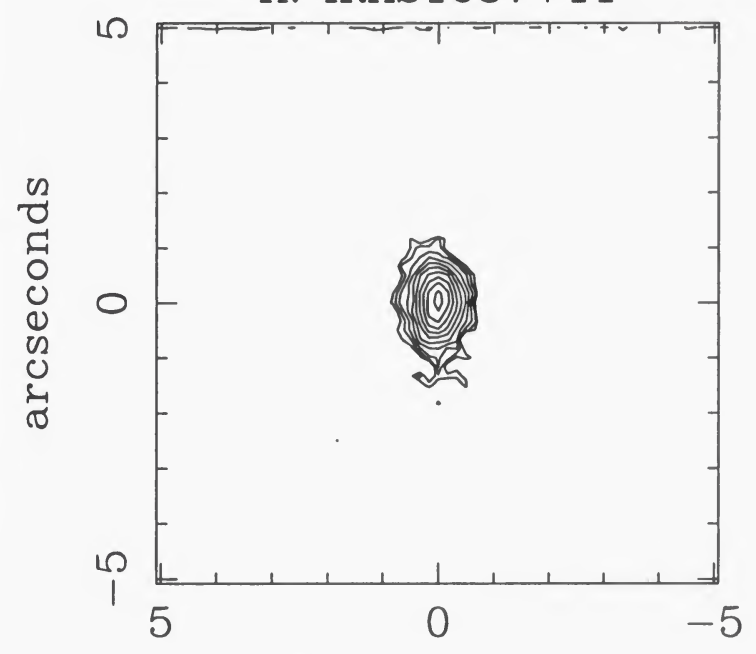

K: IRAS1049+44

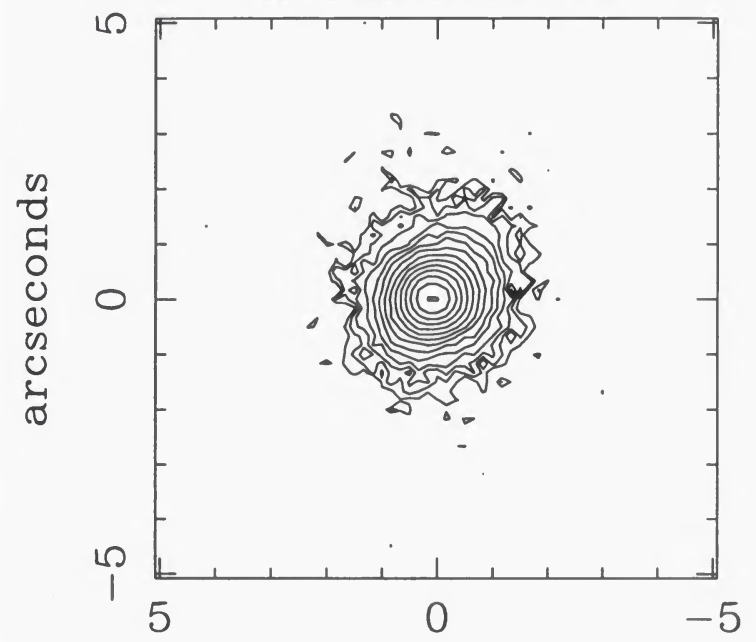

K: IRAS1056+24

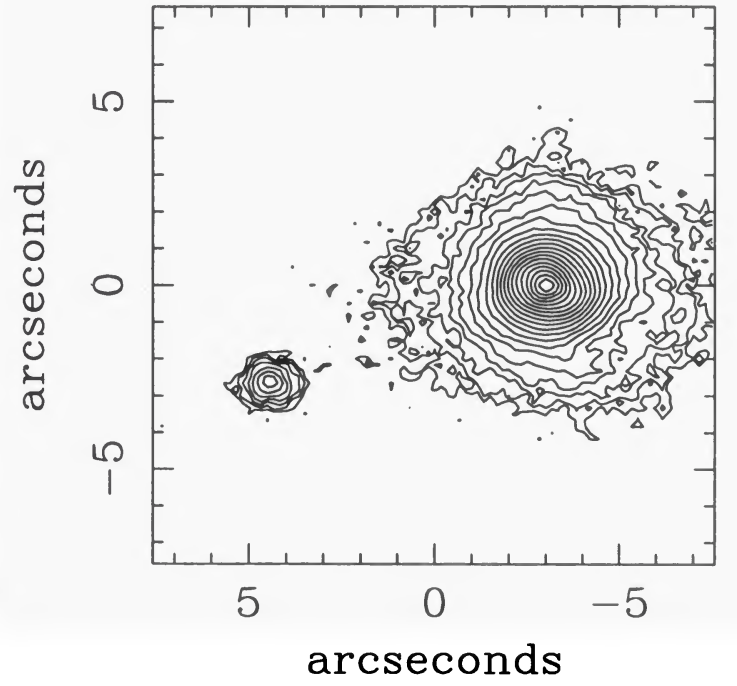

r: IRAS1037+11

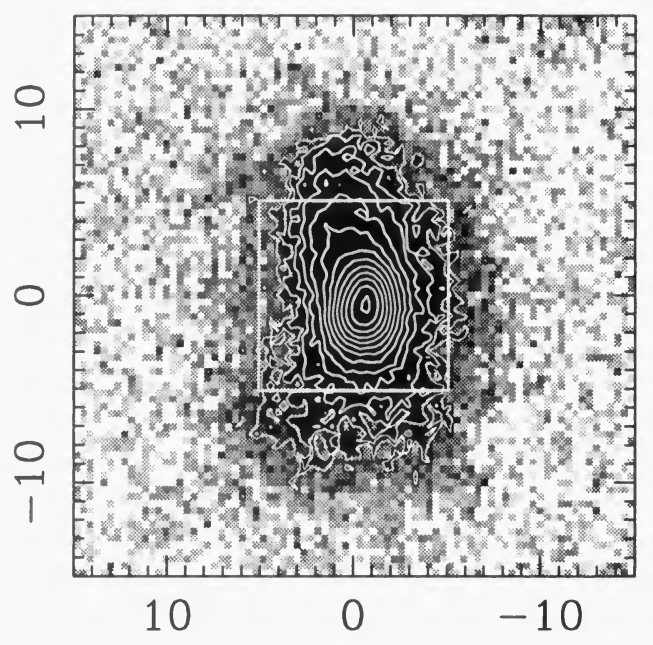

r: IRAS1049+44

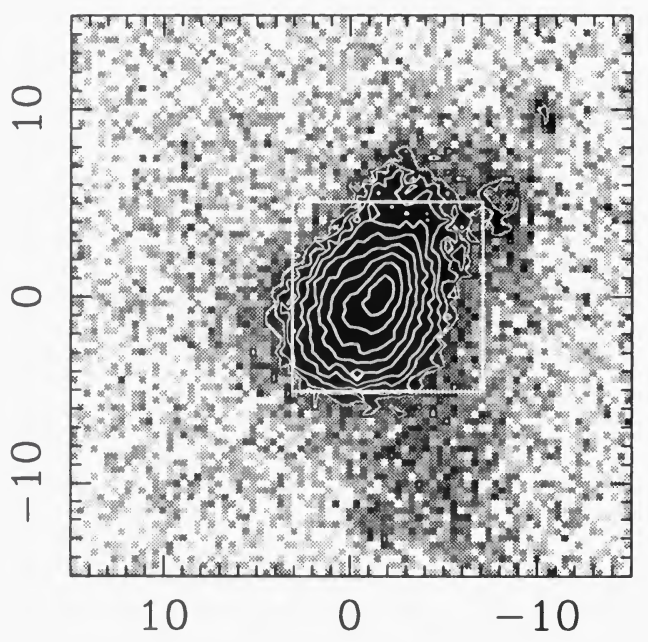

$\mathrm{r}:$ IRAS $1056+24$

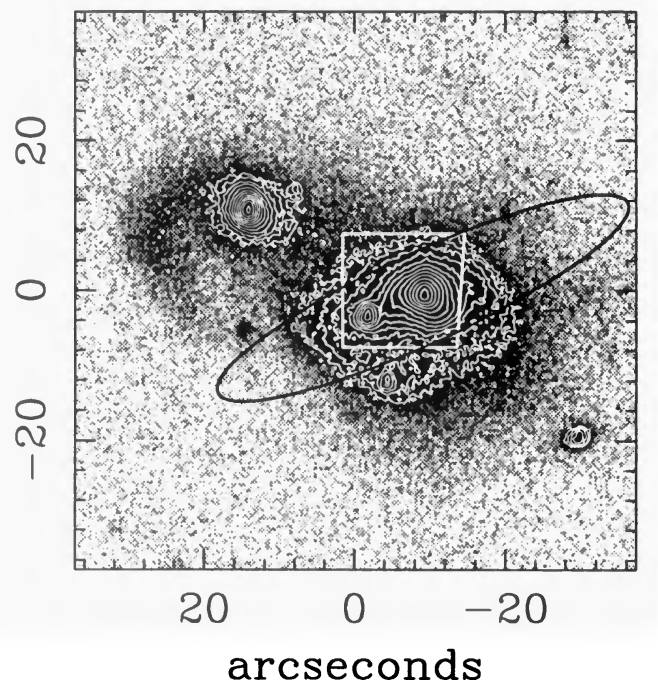

FIG. 1. (continued) 

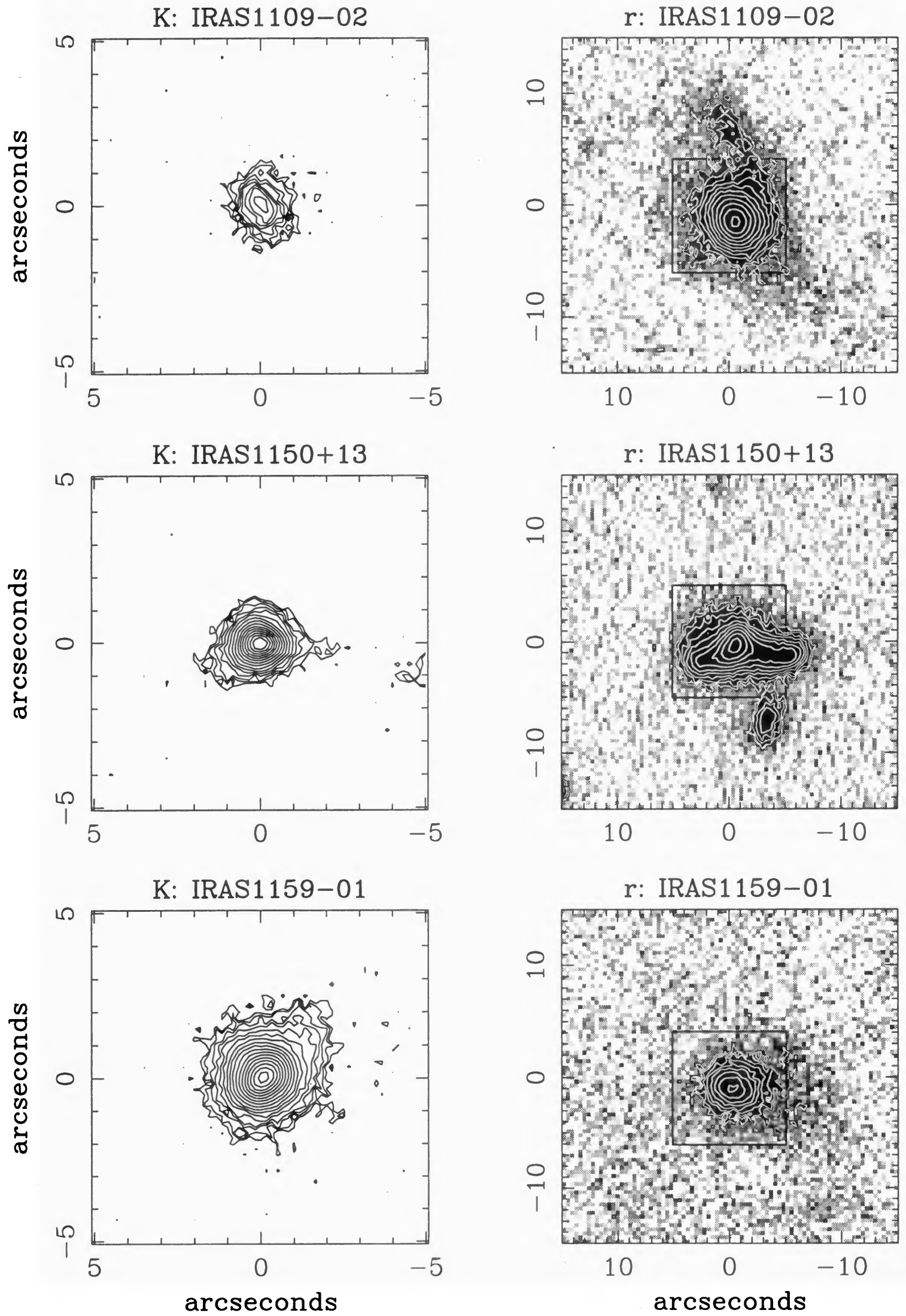

FIG. 1. (continued) 

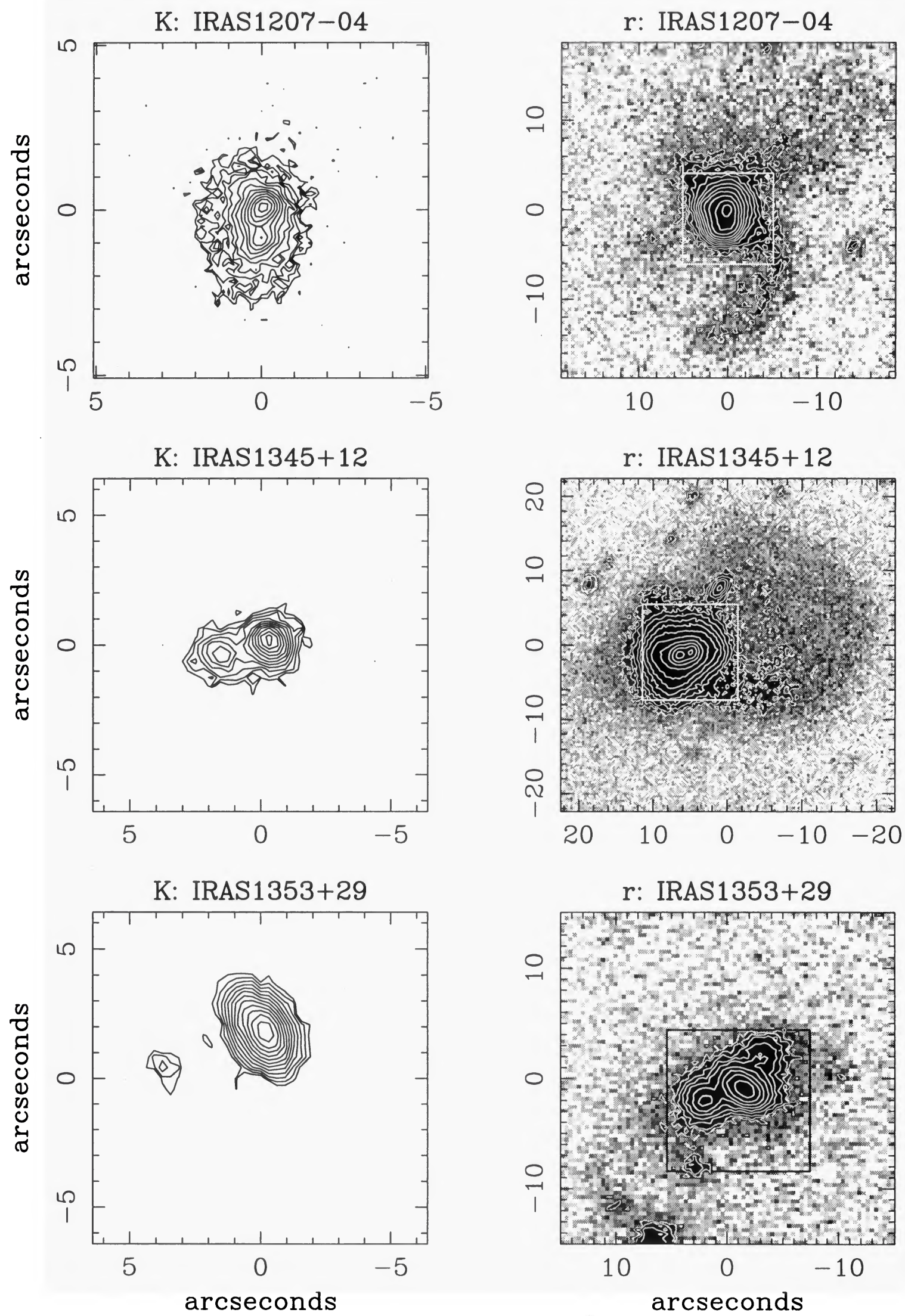

FIG. 1. (continued) 

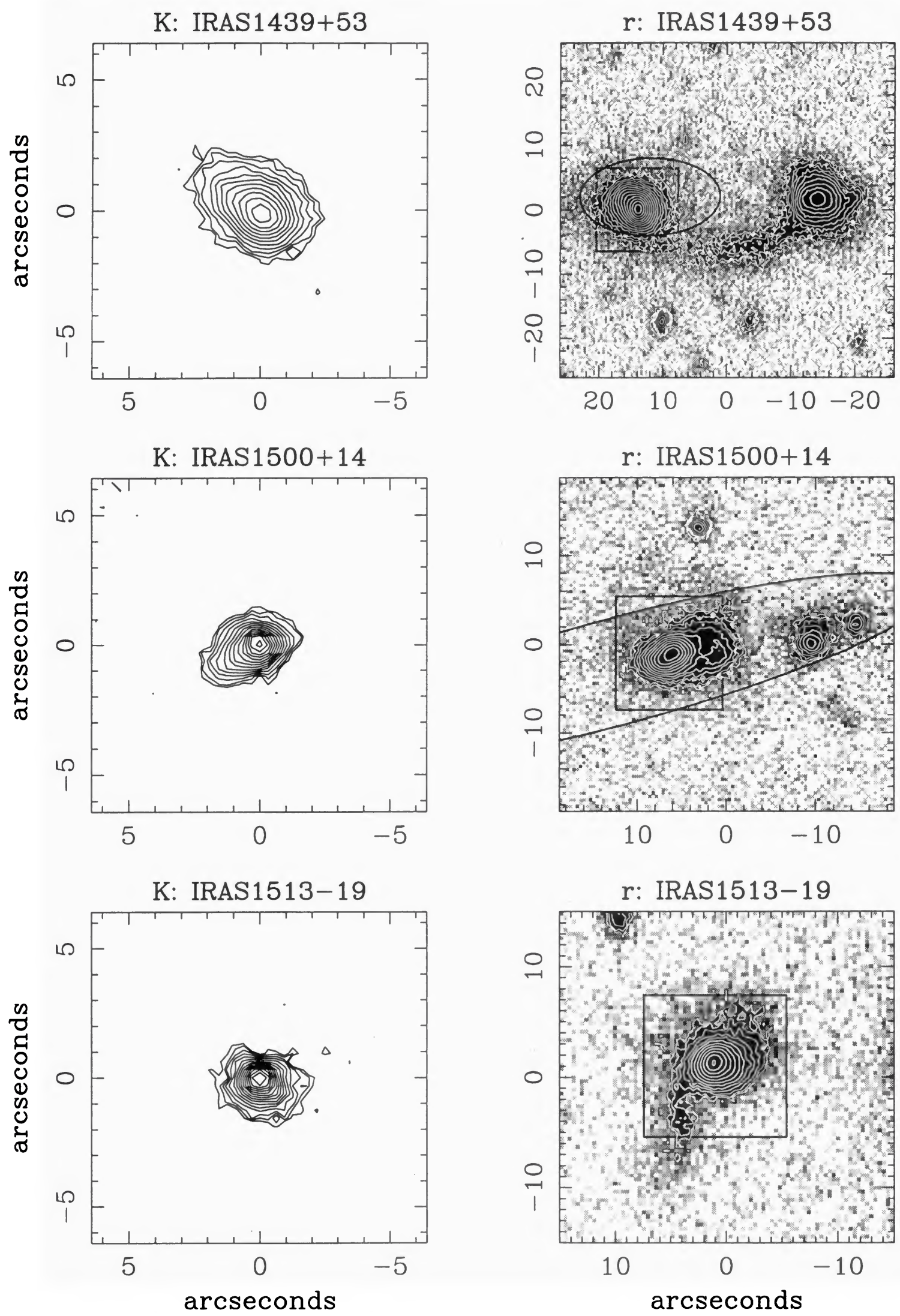

FIG. 1. (continued) 

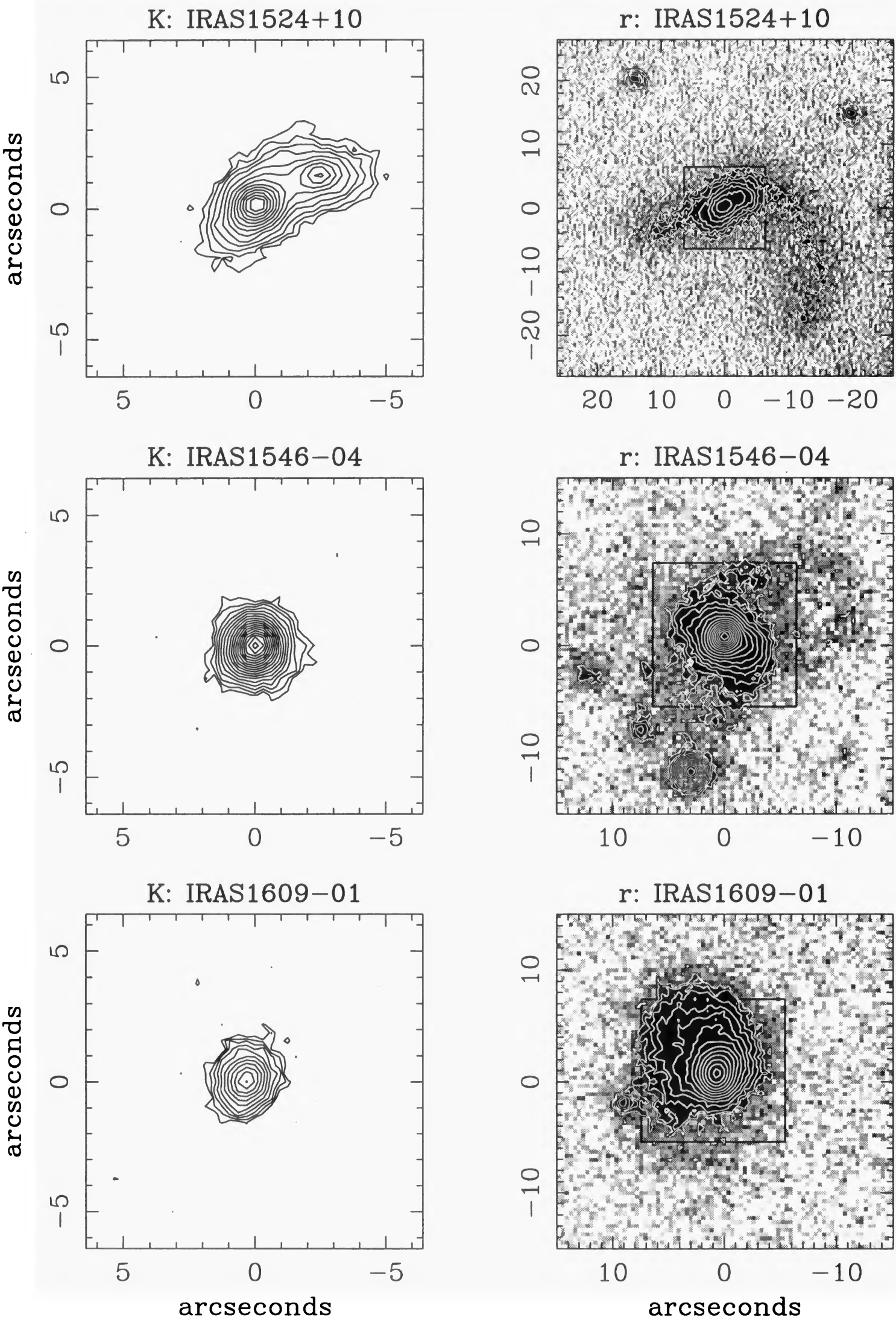

Fig. 1. (continued) 

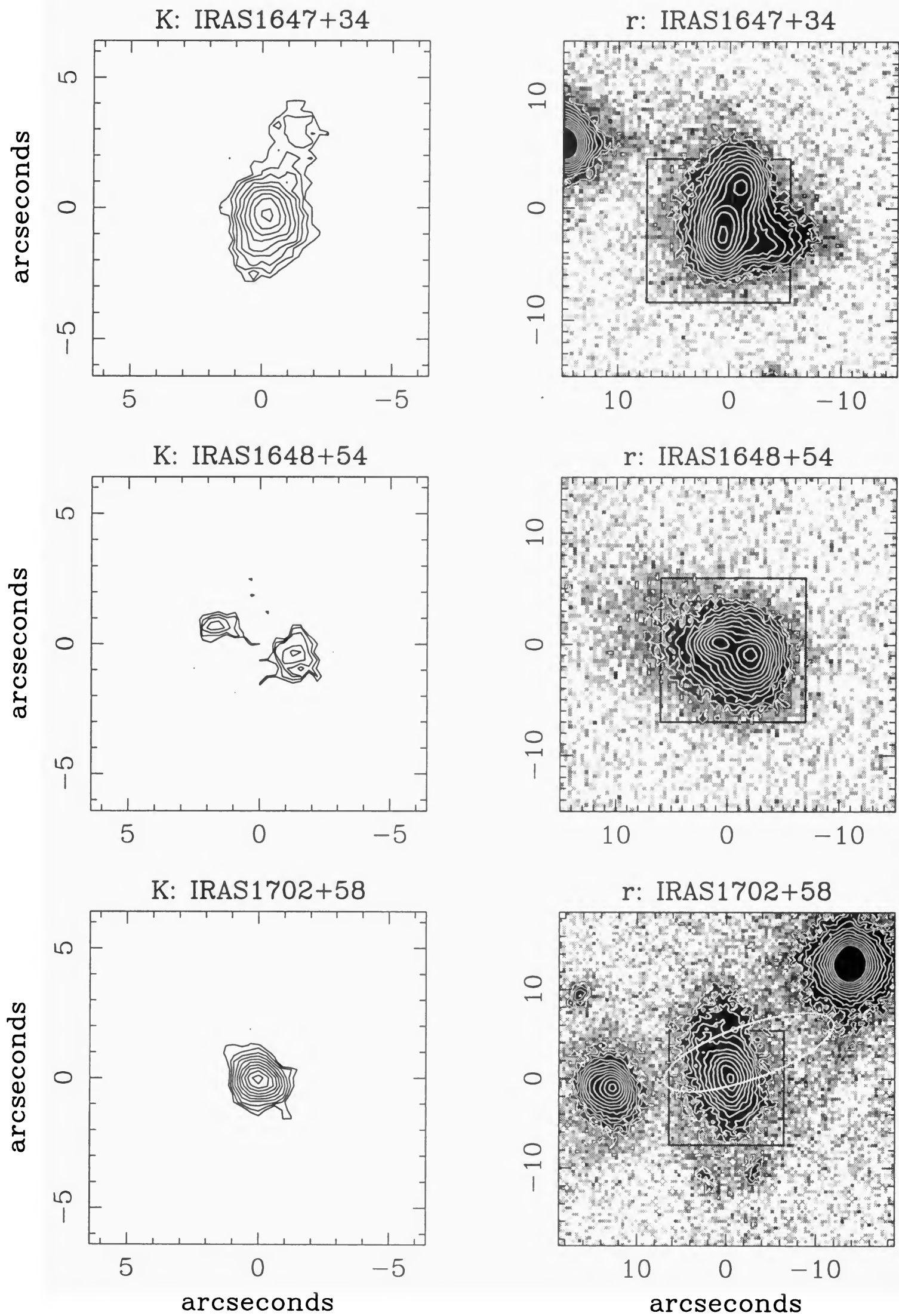

FIG. 1. (continued) 

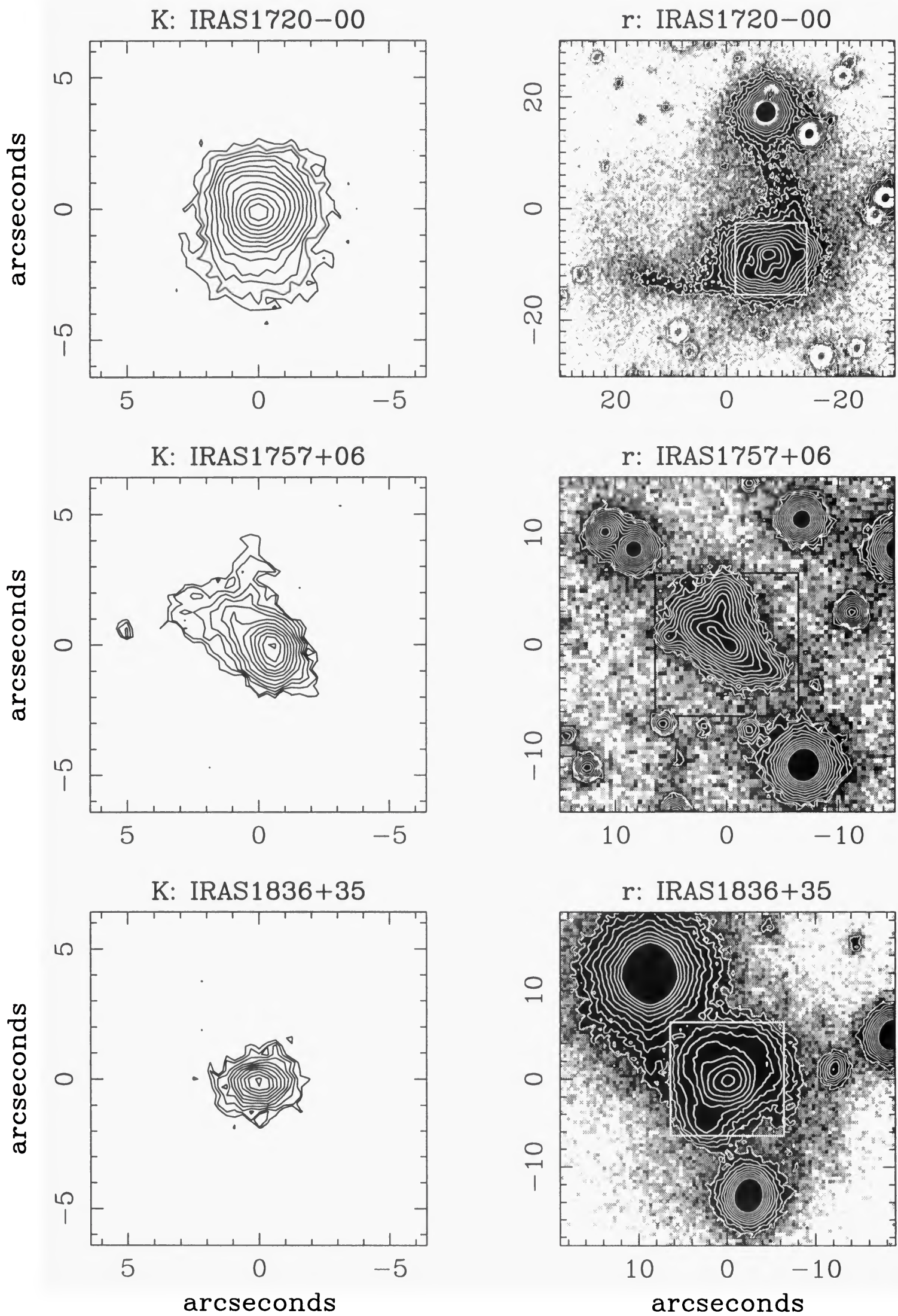

FIG. 1. (continued) 

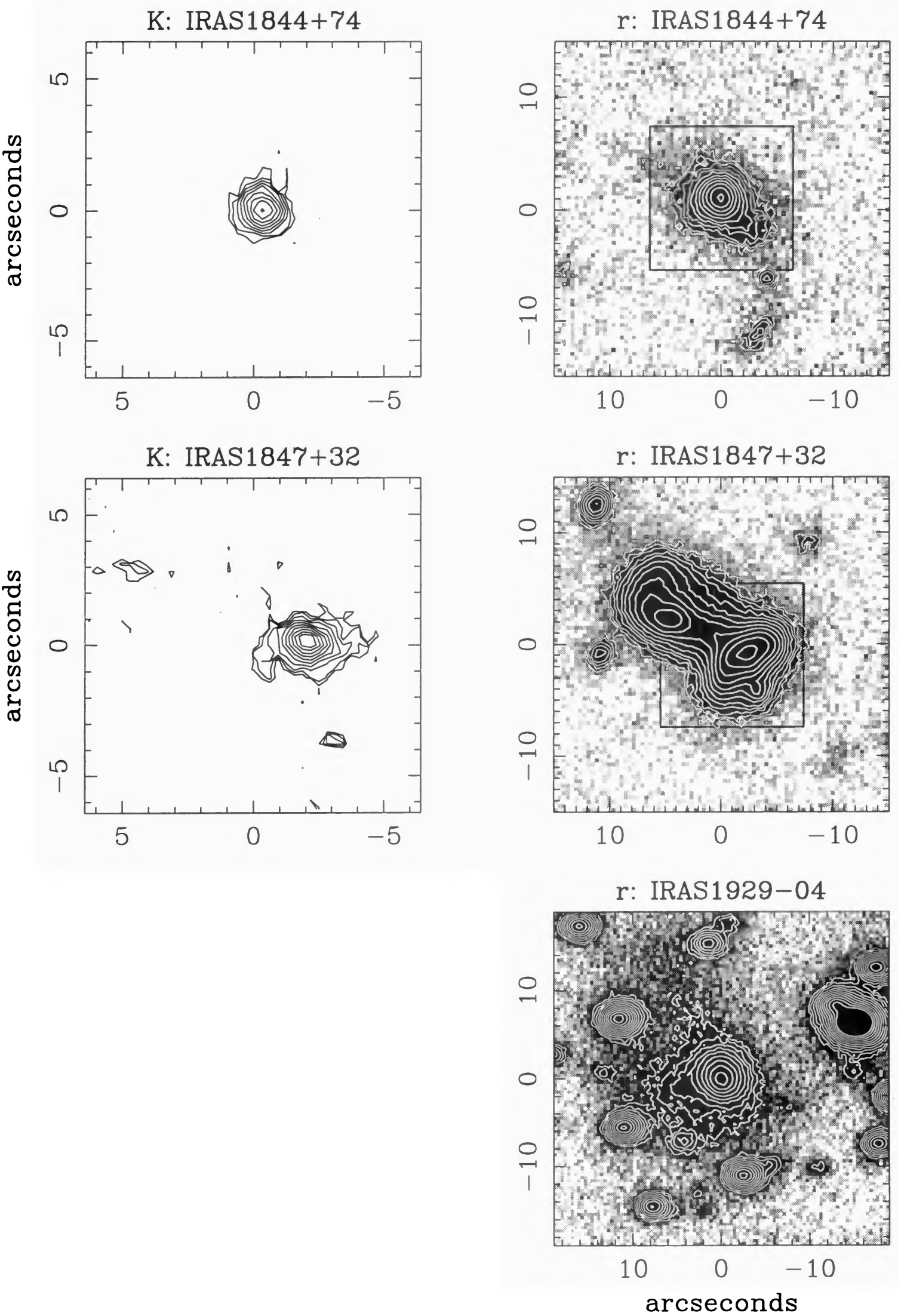

FIG. 1. (continued) 

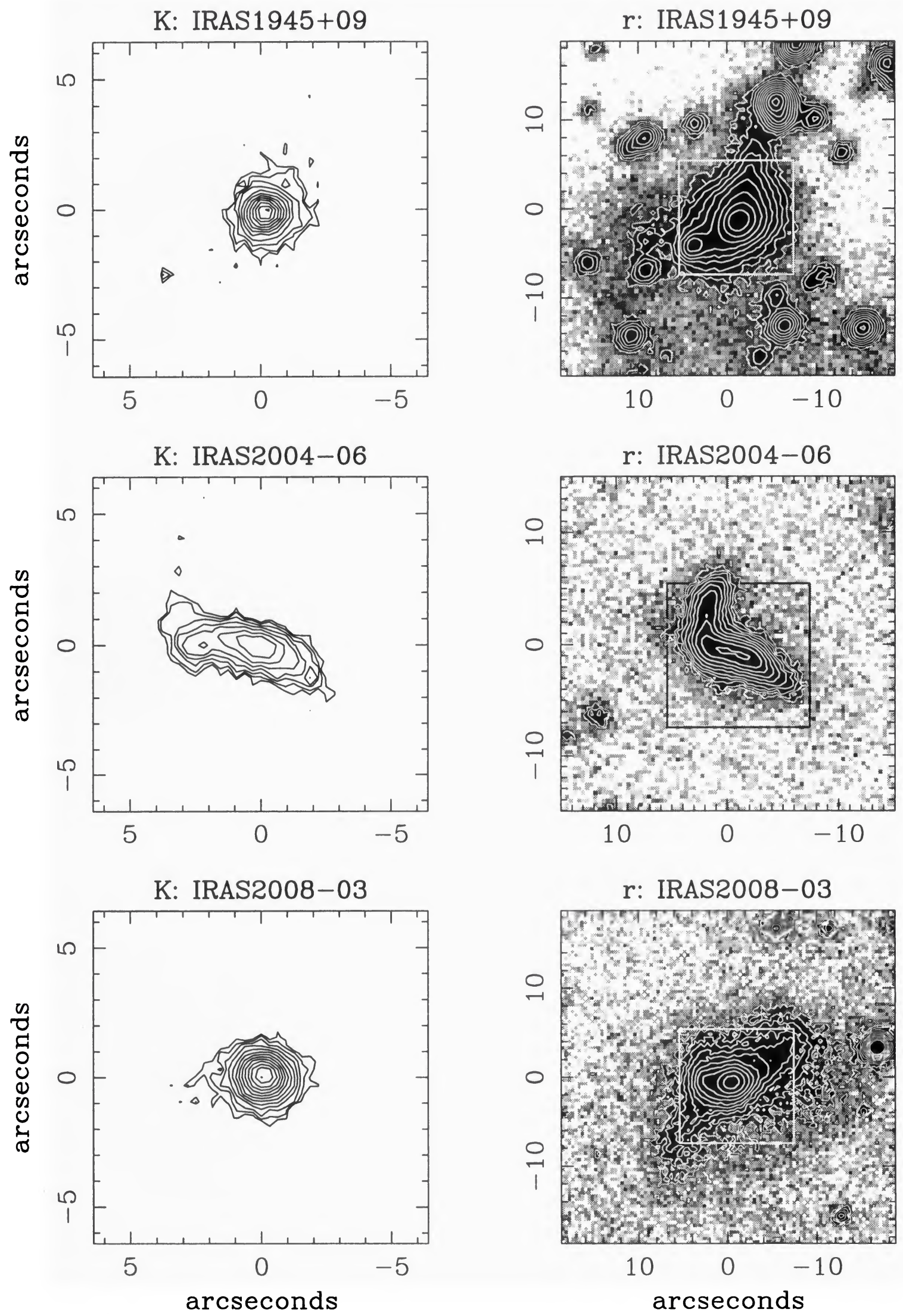

FIG. 1. (continued) 

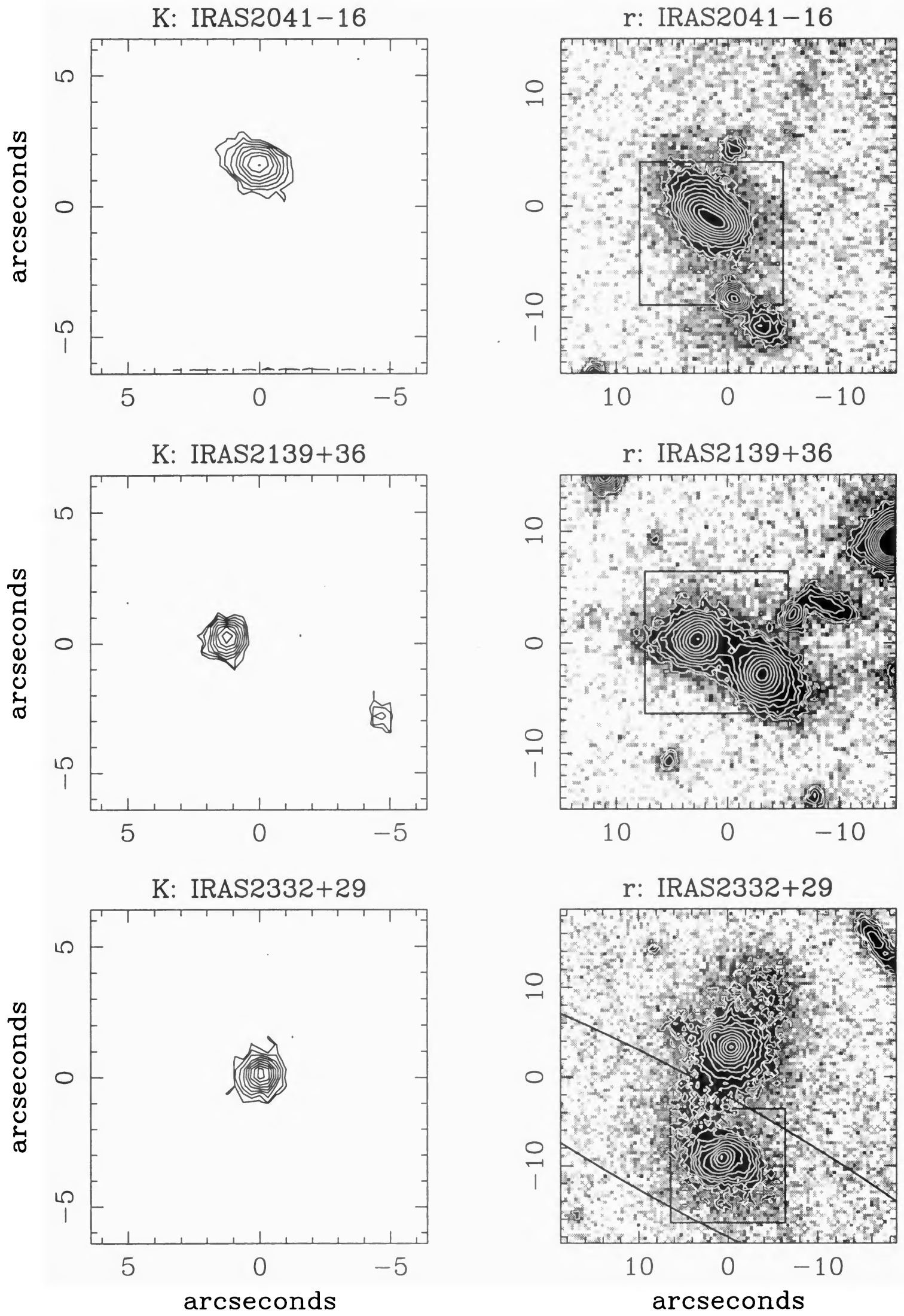

FIG. 1. (continued) 

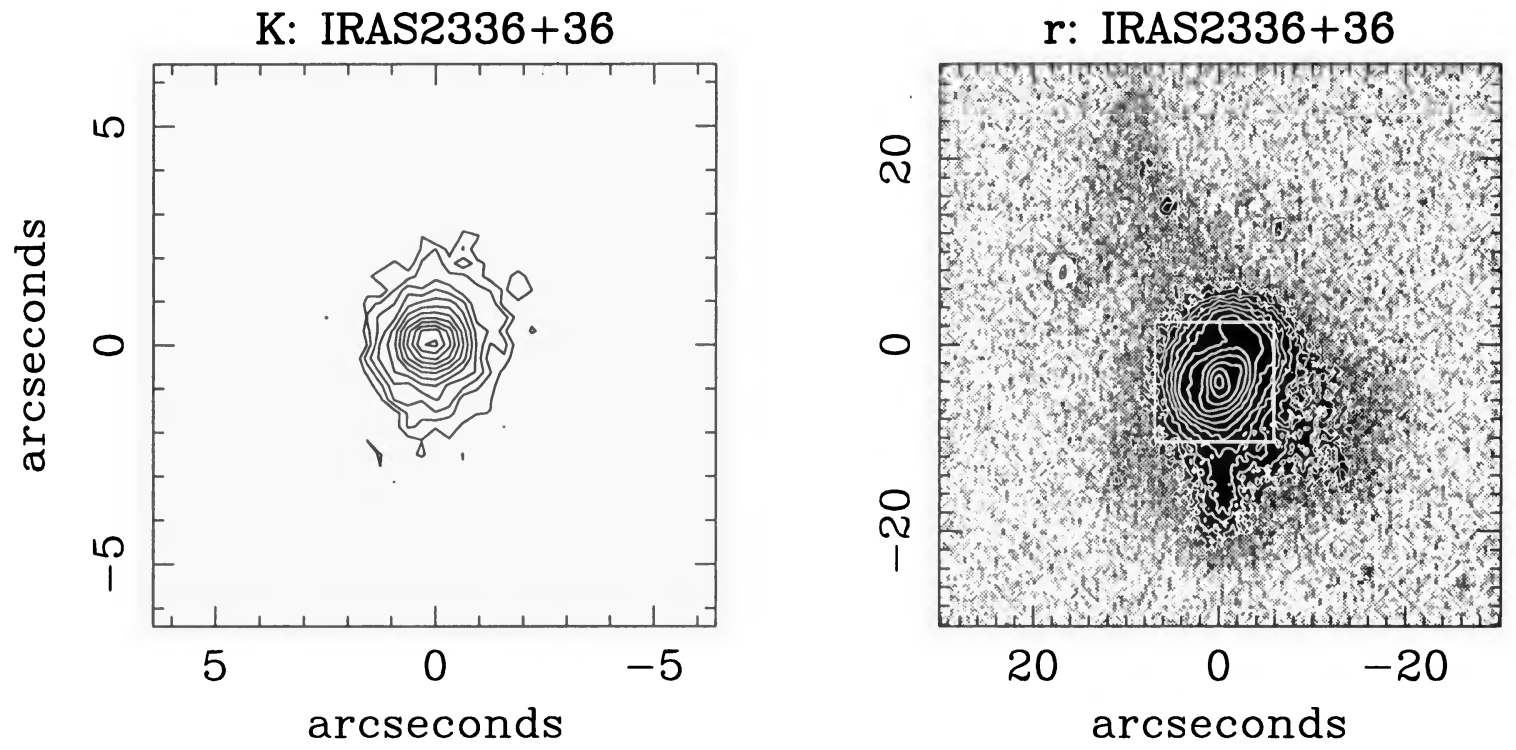

FIG. 1. (continued)

galaxies are visible, but not obviously interacting. However, in this system, the two galaxies have velocities within 165 $\mathrm{km} \mathrm{s}^{-1}$ of each other, and will thus most likely merge. The subset of double nucleus systems does not include the galaxies IRAS 03158+4227, IRAS 09111-1007, and IRAS $15001+1433$. These galaxies have a nearby, resolved component which is not apparently interacting with the primary galaxy. The case of IRAS $15001+1433$ is discussed further in Appendix A. Further observations may show these systems to be merging, but for now we classify them as single systems.

Among the double nucleus systems, there is a large spread in measured nuclear separations. The closest pair (Arp 220) has a projected nuclear separation of only $0.3 \mathrm{kpc}$, while the most widely separated pair (IRAS $14394+5332$ ) has a projected nuclear separation of $48 \mathrm{kpc}$. Companions in systems with the largest separations are visible only in the $r$ band frames due to the substantially smaller field of view of the infrared cameras.

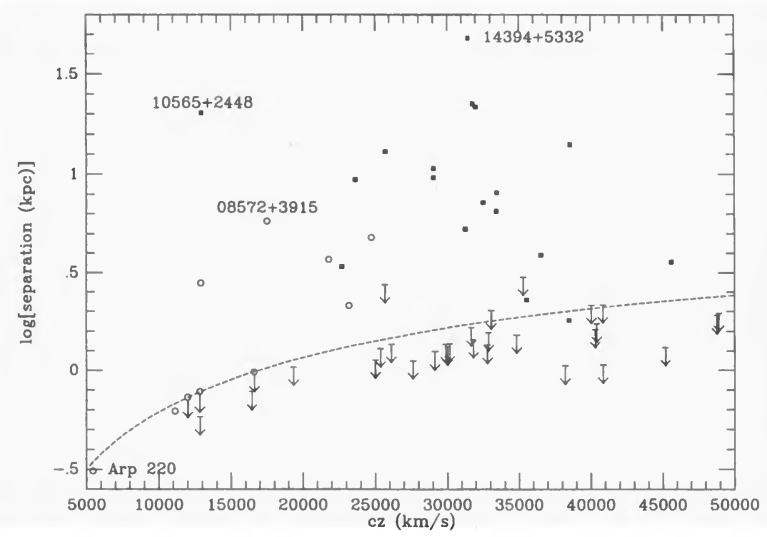

FIG. 2. Projected nuclear separation as a function of redshift for the $562 \mathrm{Jy}$ ULIRGs with available imaging data. Open circles denote the ten ULIRGs in the BGS. Points with downward arrows denote ULIRGs with single apparent nuclei, placing upper limits on the separations of double nuclei in these systems. The dashed line is the projected physical separation corresponding to one arcsecond as a function of redshift.
The nuclear separations are plotted against the recessional velocity in Fig. 2 for the 56 galaxies in the 2 Jy sample with available imaging data. Arp 220 and IRAS 08572+3915, the BGS ULIRGs with the smallest and largest projected nuclear separations, respectively, are individually labeled, as are IRAS $10565+2448$ and IRAS $14394+5332$ (see below). In this figure, the ULIRGs with single nuclei are plotted with upper limits on their nuclear separations. The dashed line represents the projected physical separation for two nuclei having an angular separation of one arcsecond.

In Fig. 3 the distribution of nuclear separations is plotted in histogram form. Here, for clarity, the 2 Jy ULIRGs with available imaging data are divided into three histograms. The first [Fig. 3(a)] shows only the 46 faint sample galaxies imaged in this paper, the second [Fig. 3(b)] shows the ten galaxies in the BGS, and the third [Fig. 3(c)] shows both groups combined. It is obvious from Fig. 3(c) that there are a large number of apparently single nucleus systems as well as a significant number of interacting pairs with wide separations. The 2 Jy sample contains seven galaxies with measured separations larger than $10 \mathrm{kpc}$. In contrast, none of the ten BGS ULIRGs selected by Sanders et al. (1988a) have projected separations greater than $10 \mathrm{kpc}$. The original BGS sample does contain one ULIRG, IRAS $10565+2448$, with a measured separation of more than $10 \mathrm{kpc}$, which was not a
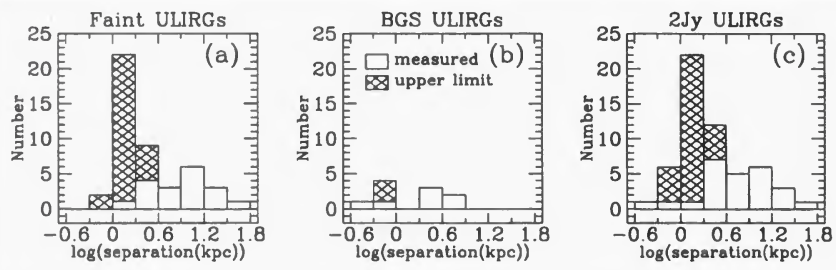

FIG. 3. Histograms of the logarithm of the projected linear separation (in kpc for the 2 ly ultraluminous infrared galaxy sample. In (a) we show data for the 462 ly ULIRGs imaged here, while (b) shows the ten BGS galaxies previously studied, and (c) combines the two, showing all 562 ly ULIRGs with available imaging data. The cross-hatched bars represent the galaxies

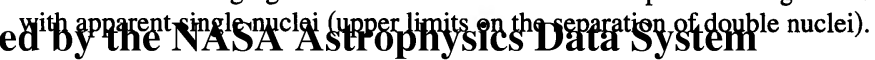


TABLE 3. 2 Jy ULIRGs in the BGS.

\begin{tabular}{lccccccc}
\hline \hline Object Name & $\begin{array}{c}\text { Other } \\
\text { Names }\end{array}$ & $\begin{array}{c}\text { Infrared } \\
\text { Morphology }\end{array}$ & $\begin{array}{c}\text { Optical } \\
\text { Morphology }\end{array}$ & sep. (") & sep. (kpc) & m $_{\mathbf{r}}$ & $\mathrm{M}_{\mathrm{r}}$ \\
\hline IRAS 05189-2524 & & $\mathrm{S}$ & $\mathrm{St}$ & $<1.0$ & $<0.8$ & 14.6 & -21.8 \\
IRAS 08572+3915 & & $\mathrm{D}$ & $\mathrm{D}$ & 5.6 & 5.8 & 14.1 & -23.1 \\
IRAS 09320+6134 & UGC 05101 & $\mathrm{S}$ & $\mathrm{St}$ & $<1.0$ & $<0.7$ & 14.3 & -22.0 \\
IRAS 12112+0305 & & $\mathrm{D}$ & $\mathrm{S}$ & 2.9 & 3.7 & 15.8 & -22.0 \\
IRAS 12540+5708 & Mrk 231 & $\mathrm{D}$ & $\mathrm{St}$ & 3.5 & 2.8 & 12.2 & -24.2 \\
IRAS 13428+5608 & Mrk 273 & $\mathrm{D}$ & $\mathrm{St}$ & 0.9 & 0.6 & 13.6 & -22.5 \\
IRAS 14348-1447 & & $\mathrm{D}$ & $\mathrm{D}$ & 3.4 & 4.8 & 15.7 & -22.4 \\
IRAS 15250+3609 & & $\mathrm{S}$ & $\mathrm{S}$ & $<1.0$ & $<1.0$ & 15.3 & -21.7 \\
IRAS 15327+2340 & Arp 220 & $\mathrm{D}$ & $\mathrm{St}$ & 0.9 & 0.3 & 13.2 & -21.2 \\
IRAS 22491-1808 & & $\mathrm{D}$ & $\mathrm{St}$ & 1.6 & 2.1 & 15.8 & -22.1 \\
\hline
\end{tabular}

Col. 1: IRAS designation.

Col. 3: Morphological classification of the $K$ band images, obtained from Carico et al. (1990), Graham et al. (1990), Majewski et al. (1993), and Armus et al. (1994).

Col. 4: Morphological classification of the $r$ band images, from Sanders et al. (1988a).

Col. 5: Separation of interacting pairs, measured in arcseconds from the $K$ band images of Carico et al. (1990), Graham et al. (1990), Majewski et al. (1993), and Armus et al. (1994).

Col. 6: Projected separation of pairs in kiloparsecs, obtained from the values in col. 5.

Col. 7: Apparent $r$ band magnitudes from Sanders et al. (1988a), with a 50 arcsec beam.

Col. 8: Absolute $r$ band magnitudes, calculated from the values in col. 7 as described in the text.

member of the group of galaxies studied by Sanders et al., although it has a $60 \mu \mathrm{m}$ flux density of $12.17 \mathrm{Jy}$ and a Galactic latitude of $64^{\circ}$, and thus meets all the criteria for inclusion in the BGS. It has a nuclear separation of 25.8 arcsec, or $20.2 \mathrm{kpc}$. With the exception of IRAS $10565+2448$, the galaxies imaged here with wide separations represent a tail in the distribution not identified previously in the brighter members of the ULIRG class.

In determining the number of 2 Jy ULIRGs with double nuclei, it is important consider the possibility that some of these galaxy pairs are in fact chance superpositions of field galaxies. We have restricted the list of double nucleus systems to those galaxy pairs which appear to be physically interacting, i.e., those with stellar bridges and tails, or common stellar envelopes. We therefore estimate the number of overlapping galaxy pairs due to chance superposition expected in the 2 Jy ULIRG sample. The typical field spiral has a $B-r$ color of $\sim 0.75$ mag (Kirshner et al. 1978, 1979; Kent 1985: Thuan \& Gunn 1976). The $B$ band magnitude of a field spiral with $r=16.5 \mathrm{mag}$ (the average $r$ band magnitude of the faint ULIRGs) is then $B=17.2 \mathrm{mag}$. The galaxy counts of Kirshner et al. suggest there are $1.26 \times 10^{0.58(B-15)}$ galaxies $\mathrm{deg}^{-2} \mathrm{mag}^{-1}$. Integrating this over $19>B>15 \mathrm{mag}$ (to account for field galaxies somewhat brighter or fainter than the typical 2 Jy ULIRG), and over a circular patch of sky with radius 11.5 arcsec $(10 \mathrm{kpc}$ at the average distance of the 2 Jy galaxies) yields only a $0.6 \%$ chance of finding an overlapping field spiral for a particular (average) ULIRG. For the entire 2 Jy ULIRG sample we would then expect less than one such occurrence. Chance superpositions of field spirals are thus not a significant problem in this sample.

Comparison of the $r$ band and $K$ band images highlights the complementary physical scales probed by the two wavelengths. At visible wavelengths the detection of faint, low surface brightness extended structure is much easier, therefore deep visual images are crucial for a proper estimate of the fraction of the sample galaxies which show evidence for interactions. The $K$ band frames, on the other hand, are valuable for revealing the locations of the stellar mass concentrations within the dusty environments at the centers of the galaxies. The positions and relative brightnesses of the nuclei can be used to estimate the time until merger and lifetime of the ultraluminous phase (see Sec. 4). Some galaxies (e.g., IRAS 00091-0738, IRAS 03521+0028, and IRAS 15245 +1019 ) have clear double nuclei in the $K$ band which are invisible in the $r$ band. The discrimination of nuclei from hotspots and or holes in the obscuration (e.g., as in Arp 220), although unavoidably subjective without multiwavelength spectroscopic information, is made simpler through the inclusion of both visual and near-infrared images.

\subsection{Visual Magnitudes}

Apparent $r$ band magnitudes (Hayes \& Latham 1975; Thuan \& Gunn 1976) for all galaxies observed under photometric conditions are presented in Table 2. Galactic extinction corrections, derived from the H I maps of Burstein \& Heiles (1982), have been applied. The $r$ band images typically have $3 \sigma$ sky levels of 23.5-24.0 mag $\operatorname{arcsec}^{-2}$, yet inspection of Fig. 1 shows that detail is visible in each of the galaxies at levels much fainter than this (i.e., fainter than the lowest contour). In order to obtain an accurate measurement of the total magnitude of each system, we have used apertures large enough to include the faint structure associated with each galaxy, but without including flux from stars or other galaxies in the field. Three of the galaxies (IRAS $00153+5454$, IRAS 08311-2459, and IRAS 19458+0944) are at low Galactic latitudes $\left(|b|<10^{\circ}\right)$ and the foreground extinction corrections are therefore both large and uncertain. For these galaxies, we have estimated the Galactic extinction to be $\mathrm{E}(B-V)=0.25 \mathrm{mag}$, by extrapolation from the $\mathrm{H}$ I maps of Burstein \& Heiles. For systems containing widely separated galaxies we quote the $r$ band magnitude only for the galaxy thought to contribute to the far-infrared flux as determined by the IRAS positional uncertainty ellipses. The error ellipses, representing the position of the infrared source at the $95 \%$ confidence level, are plotted in Fig. 1 for systems in which two or more bright galaxies appear in the field. Included in this set are the four interacting galaxies with the largest projected physical separations. For each of these sys- 
tems we quote magnitudes for both components of the system in Table 2. Notes on the derivations of individual magnitudes are also included in Table 2. Apparent $r$ band magnitudes for the BGS galaxies (as measured by Sanders et al. 1988a), are given in Table 3.

Absolute $r$ band magnitudes, uncorrected for internal reddening, are also presented in Tables 2 and 3. These magnitudes have been computed according to the formula: $M_{r}=m_{r}-15.62-5 \log [\mathrm{cz}(1+z / 2)]-10 \log [1+z]-K$, where $K$ is the $K$ correction. We have used the Gunn $r$ band $K$ correction (typically $0.01-0.05 \mathrm{mag}$ ) taken from Sebok (1986). The average $M_{r}$ of the $2 \mathrm{Jy}$ sample is $-22.2 \mathrm{mag}$, and ranges from -21.1 to $-24.4 \mathrm{mag}$. For comparison, an $L^{*}$ galaxy (Schechter 1976), has an $M_{r}^{*}=-20.5 \mathrm{mag}$, using a typical $B-r=0.75 \mathrm{mag}$ and $M_{B}^{*}=-19.7 \mathrm{mag}$. Based on the positional uncertainties, it is unclear whether or not both galaxies in the systems IRAS $17028+5817$ and IRAS 23327 +2913 contribute to the infrared flux. Therefore the absolute magnitude used in calculating the average above represents the combined flux from the constituent galaxies for these two systems. For all other cases where ellipses are drawn in Fig. 1 , only the galaxy or galaxies within the ellipse are included in the calculation of the average absolute magnitude. The 2 Jy ULIRGs therefore have absolute $r$ band magnitudes which range from 0.5 to 3.9 mag more luminous than $M_{r}^{*}$, and thus they inhabit the tail of the luminosity function for normal galaxies. Corrections for internal extinction would likely drive the $2 \mathrm{Jy}$ ULIRGs further from $M_{r}^{*}$, as the ULIRG selection criteria favor galaxies that are more dusty than normal galaxies.

\section{DISCUSSION}

It is obvious from Fig. 1 that the 2 Jy ULIRGs, though selected for their high infrared luminosities, form a morphologically diverse group. The large fraction of galaxies with distorted morphologies, along with the large number of double nucleus systems provides compelling evidence that the ultraluminous infrared galaxies are in the process of merging, and by inference, that this merger has triggered the ultraluminous phase of the system. With this in mind, the images of the sample galaxies can be thought of as "snapshots" of the merger sequence within the ultraluminous phase. If the time over which the ULIRGs remain infrared bright is at least as long as the time required for the galaxies to complete their merger, then the average time until merger in a large sample of merging ULIRGs, which can be inferred from imaging data, can be used to estimate the lifetime of the ultraluminous phase.

In a galactic interaction, merger is brought about by the transfer of orbital energy to internal degrees of freedom in the systems through dynamical friction. This process is greatly facilitated by the presence of massive, dark halos surrounding the visible disks (see Barnes \& Hernquist 1992 for a review). Although dynamical friction between the luminous parts of the systems is most effective at the smallest radii, the galaxies spend relatively little time there compared to the total time scale of the interaction. The net angular momentum of the nuclei drops significantly at each passage, and after only a few close passes, the orbits circularize and the two nuclei spiral together. The gas in the galaxies also loses its angular momentum and becomes concentrated in the central region of the remnant, where it can fuel a starburst, an AGN, or both. The short wavelength radiation produced in the active region is absorbed and reradiated in the farinfrared by the surrounding dust. Because the galaxies spend very little time at close separation in the early stages of the interaction, a sampling of interacting/merging galaxies not selected for extreme luminosity would yield few systems at close separation. The majority of galaxies in the 2 Jy ULIRG sample, in contrast, have separations much less than $10 \mathrm{kpc}$, indicating that these selection criteria preferentially favor the choice of galaxies at very close separation and in the final stages of merging.

The sequence of events we envision in the evolution of a ULIRG starts with the interaction of a bound pair of spiral galaxies which approach each other on nearly parabolic orbits. Distorted morphologies resulting from the tidal interaction become apparent early, typically occurring around the time of initial closest approach. Due to an increase in the central gas density and the number of cloud-cloud collisions, a massive starburst can be triggered. We assume that the system becomes ultraluminous at some time after initial closest approach, and that this phase lasts at least as long as the remaining merger lifetime. Finally, the nuclei spiral in toward each other and merge. We assume here that all ULIRGs are the product of interactions between large, gas-rich galaxies (recall that $95 \%$ of the ULIRGs in this sample exhibit disturbed morphologies). It is important to note that although current models of merging gas-rich galaxies favor the generation of an intense starburst as the source of the ultraluminous power, the dynamical time scale estimates which follow do not depend on the exact nature of the power source.

Based on the imaging data presented here, once the system becomes ultraluminous, three dynamical stages can be identified. The first stage corresponds to the time after "turn on" while the nuclei are still well separated, as in IRAS $14394+5332$. The orbits in this stage can be described as predominantly radial. The second stage is one in which the galaxies are very close with overlapping luminous disks, and the clearly distinct nuclei of the galaxies are essentially spiraling in toward each other on nearly circular orbits (e.g., IRAS $13451+1232$, IRAS $15245+1019)$. The third stage is simply the time spent after final merger while the system is still ultraluminous in the infrared. The apparently single system, IRAS 17208-0014, may represent this stage. In this model the ultraluminous phase lasts at least as long as the time required for the merger to be completed. This seems to be a reasonable assumption given that so many of the ULIRGs appear as unresolved, but, morphologically distorted objects (i.e., some may be post-merger systems).

For the purposes of this discussion, we assume that a merging ULIRG spends its life in the first stage until the distance between merging nuclei becomes less than $10 \mathrm{kpc}$. At this distance the disks are likely to overlap and the orbits will circularize causing the nuclei to rapidly merge. It is important to note that for an individual galaxy all we can estimate from the images and these simple dynamical argu- 
ments is the time from the present until the time of final merger, when the nuclei of the interacting galaxies coalesce. We must rely on the distribution of these time scales within the sample, along with the morphologies of the galaxies, to allow a calculation of the lifetime of the ultraluminous phase. Thus we begin the following calculations by estimating the time until final merger for each sample galaxy. For galaxies which are presently at very large separations, the time remaining in the first stage is calculated, and then added to the estimated time to be spent in the second stage. For galaxies which are presently at small separations, only the time remaining in the second stage is calculated.

The time remaining in the first stage can be estimated by assuming that the nuclei approach nearly radially at a velocity $V_{r}$ until the nuclei are $10 \mathrm{kpc}$ apart. Because of the small range in nuclear separations, and the large uncertainties involved, we treat the radial velocity $V_{r}$ as constant. In this case, the time from observation until the nuclei are within 10 $\mathrm{kpc}$ is $(r-10 \mathrm{kpc}) / V_{r}$, where $r$ is the nuclear separation. The time spent in the second stage can be estimated as $(\mathscr{L} / m) \times(2 \pi r) / V_{c}$, where $\mathscr{C l} / m$ is the mass ratio of the nuclei, $r$ is the nuclear separation, and $V_{c}$ is the orbital velocity (E. S. Phinney, private communication). This is essentially the dynamical time scale times the mass ratio since, as the nuclei spiral together, the relative velocity is well approximated by the circular velocity at that radius (Binney \& Tremaine 1987). If we assume that ULIRGs "turn on" randomly in time, and that they remain ultraluminous at least until final merger, then the time spent in the pre-merger stage for an individual ULIRG is, on average, twice the time it will take to merge from its current state. This is simply a consequence of random sampling of events occurring uniformly in time. Finally, there is the time spent in the post-merger stage. This stage lasts as long as the galaxy remains an ultraluminous infrared system beyond final merger. Although the time from observation until merger may be computed for the double nucleus ULIRGs with measurable separations, the majority of the sample galaxies are apparently single. These ULIRGs may simply be unresolved doubles, or true postmerger systems. We investigate the two limiting cases in which all of the unresolved ULIRGs are either single or all are double in Secs. 4.1 and 4.2, to provide an upper and a lower limit, respectively, on our estimates of the ultraluminous phase lifetime. These two cases suggest that the average lifetime of the ultraluminous phase lies between $2 \times 10^{8}$ and $20 \times 10^{8} \mathrm{yr}$.

In the calculations that follow we set the nuclear mass ratio equal to the $K$ band flux ratio of the nuclei. In four of the galaxies where we do not have $K$ band data on both nuclei (the large separation systems), we take the ratio of the nuclear $r$ band fluxes as an estimate of the mass ratio. The $K$ band flux ratios are typically around $2: 1$, though some are as high as 6:1. Finally, we adopt an average radial velocity $V_{r}$, and an average circular velocity $V_{c}$, of $200 \mathrm{~km} \mathrm{~s}^{-1}$ for all the double nucleus galaxies in the sample.

\subsection{Ultraluminous Lifetime-Unresolved Singles}

One approach for estimating the lifetime of the ultraluminous phase is to assume that all the unresolved ULIRGs are post-merger systems. The ratio of time spent in the postmerger phase to the time spent in the pre-merger phase is then equal to the ratio of the number of single systems to the number of double systems $\left(N_{\text {single }} / N_{\text {double }}\right)$. Of the 53 interacting galaxies in the 2 Jy sample for which imaging data are available, $25(47 \%)$ have double nuclei. With a measurement of the $K$ band flux ratio and the projected separation of each of the double nucleus systems, we can estimate the time until merger. Using the logarithm of the time until merger as the independent variable, the mean time until merger for the measured double nucleus systems is $\left\langle T_{\text {merge }}\right\rangle$ $=5(+17,-4) \times 10^{8} \mathrm{yr}$, where the limits correspond to the sample standard deviation. The lifetime of the ultraluminous phase is then $2\left\langle T_{\text {merge }}\right\rangle \times N_{\text {total }} / N_{\text {double }}=17(+55,-13) \times 10^{8}$ yr. Using the median of the time until merger instead of the mean of the logarithmic distribution yields a value for the lifetime of the ultraluminous phase of $16 \times 10^{8} \mathrm{yr}$. Applying the same analysis to the ten BGS ULIRGs, seven of which are measured double nucleus systems, yields an average lifetime of the ultraluminous phase of $5 \times 10^{8} \mathrm{yr}$. Note that we are using the logarithmic time scale distribution in our analysis instead of the linear time scale distribution as the former is more nearly Gaussian.

One problem with estimating the merger and ultraluminous infrared phase lifetimes of the 2 Jy ULIRGs in this manner comes from the fact that the 2 Jy ULIRG sample spans a relatively large range in redshift. The mean distance of the 2 Jy ULIRG sample is about twice that of the subset in the BGS studied by Carico et al. (1990). As a result of the fixed angular resolution our ultraluminous lifetime estimate is biased towards much higher values. By not resolving the close separation doubles, the mean time until merger and the fraction $N_{\text {total }} / N_{\text {double }}$ are both larger. It is not surprising then, that the estimates calculated above are larger than those obtained by looking at the nearest sample members-those in the BGS. The same effect applies to the dependence of our estimate on atmospheric seeing. Near-infrared observations at higher spatial resolution (0.5 FWHM or less) will undoubtedly resolve more close separation pairs, thereby lowering the ultraluminous phase lifetime as estimated by this technique.

\subsection{Ultraluminous Lifetime-Unresolved Doubles}

Inspection of Figs. 2 and 3 shows that the majority of the galaxies (28/53) are in fact, apparently single nucleus systems. However, through analogy with the nearby BGS systems, some fraction of these are undoubtedly unresolved doubles. To take the extreme limit, we assume that all 28 apparently single nucleus systems are in fact unresolved doubles. This is equivalent to having the ultraluminous phase end at the time of merger. Since the unresolved doubles are necessarily at very close separations and the time until final merger is very small, the calculation will therefore give us a lower limit on the lifetime of the ultraluminous phase. For the well-resolved galaxies we can estimate the time until merger as described above. For the unresolved galaxies (assumed here to be double), another technique must be applied. The $K$ band point spread functions, taken together with the infrared observations, provide an effective upper limit to the 
projected physical separations of the unresolved ULIRGs. We can use these upper limits to approximate the time until merger for these unresolved double nucleus systems.

The simplest way to determine the ultraluminous phase lifetime under the assumption that all unresolved ULIRGs are double is to assume a uniform distribution of separations between zero and the limit set by the angular resolution of the data. The average unresolved ULIRG would then have a separation of one half the physical separation corresponding to its seeing limit. Using a mass ratio of $2: 1$ and a circular velocity of $200 \mathrm{~km} \mathrm{~s}^{-1}$, we calculate the remaining time until merger for these galaxies, and take the ultraluminous phase lifetime as twice this value. Using the logarithm of the time until merger as the independent variable gives an average lifetime of the ultraluminous phase of $2(+9,-2) \times 10^{8} \mathrm{yr}$, where again, the limits represent the standard deviation of the time scale distribution. Taking the median of the time scale distribution instead of the average of the $\log$ gives about $50 \%$ of this value, or $1 \times 10^{8} \mathrm{yr}$.

The second method we can employ to estimate the lifetime of the ultraluminous phase using the unresolved galaxies as premerger systems (doubles) of unknown separation makes use of survival analysis (see Feigelson \& Nelson 1985). In this technique, a Kaplan-Meier estimate of the mean of the distribution is calculated by making use of the censored data, in this case all upper limits. Once again we assume a mass ratio of 2:1 and a circular orbital velocity of $200 \mathrm{~km} \mathrm{~s}^{-1}$ for the unresolved galaxies, thus changing the upper limit on the separation into an upper limit in the time until merger. The upper limit on projected physical separation corresponds to the seeing limit at the time of observation for each galaxy. With these upper limits and the estimated values for the well resolved galaxies comprising the distribution, application of the Kaplan-Meier technique to the logarithm of the time scale distribution yields an average ultraluminous phase lifetime of $1.7(+0.6,-0.4) \times 10^{8} \mathrm{yr}$. Here, the limits correspond to a $1 \sigma$ uncertainty in the estimate of the mean.

If the unresolved ULIRGs are all close separation doubles, then further studies at higher angular resolution should begin to resolve more of the double nucleus systems. If the number of pairs at a given separation increases with decreasing separation, then an estimate of the lifetime by either of the methods above would result in a shorter lifetime with data of higher resolution. If, on the other hand, there are fewer pairs at smaller separations, the lifetime estimates would be higher under better seeing. In either case, the lifetime estimate would not vary significantly from $2 \times 10^{8} \mathrm{yr}$.

\subsection{Concluding Remarks-Comparison With Other Time Scales}

There is evidence for both starbursts and AGN in powerful infrared galaxies. Much of the controversy concerning these galaxies centers on the fractional importance of these two energy sources both in individual objects and among the ULIRGs as a class. It is therefore worthwhile to compare our estimates of the lifetime of the ultraluminous phase based upon dynamical arguments to those based upon the rate at which fuel must be consumed to sustain the luminosity at such high levels. In addition, since outflowing winds have been suggested as the mechanism by which a luminous starburst can clear away its dust shroud and possibly reveal a previously buried AGN, the lifetime of this process is relevant as well. In particular, can the wind phase be long enough to allow the lifetime of the ultraluminous infrared phase to extend significantly beyond final merger?

Empirically, star formation has been suggested as the source of the infrared power in a large number of luminous infrared galaxies (e.g., Rieke et al. 1985; Armus et al. 1989; Condon et al. 1991; Scoville et al. 1991). In addition, recent models of merging spiral galaxies containing both stars and gas components (Mihos \& Hernquist 1994) succeed in producing large increases in the bolometric luminosity from enhanced star formation during the late stages of the mergerthe last $10 \%$ of the total interaction time, or about $10^{8} \mathrm{yr}$. The strength, duration, and onset of this starburst episode is strongly dependent upon the presence of a massive stellar bulge, which serves to disrupt the formation of a nuclear bar and thus prevent the early build up of gas around the merging nuclei. The relatively short, intense bursts generated in the models with massive stellar bulges (bulge mass $\sim 1 / 3$ disk mass) are consistent with the notion that the ultraluminous infrared phase, if modeled as a super-starburst consuming up to 10-100 solar masses of molecular fuel per year, could last for at most a few $\times 10^{8}$ yr given the measured circumnuclear fuel supplies (Condon et al. 1991; Sanders et al. 1991). These starburst time scales are similar to what we have dynamically derived assuming the single $2 \mathrm{Jy}$ ULIRGs are all unresolved doubles.

Although the average lifetime of the ultraluminous phase as calculated in Sec. 4.2 is relatively short, there are a significant number of ULIRGs with large nuclear separations. These galaxies may contain a third, unresolved nucleus from a previous encounter which is responsible for the infrared luminosity. Alternatively, they may provide direct evidence that the ultraluminous phase can occur early in the interaction. In the merger models of Mihos \& Hernquist (1994) these galaxies cannot be explained if all the starburst activity happens at late times. A range in the bulge:disk:halo mass ratios as well as variations in orbital geometries in these systems may be responsible for the range in separations we observe. However, it is equally clear that the predominance of small separation systems and the large number of apparently single nucleus galaxies implies that ultraluminous infrared activity strongly selects galaxies with small physical separations.

If some of the ULIRGs are powered by a massive black hole of about $10^{8} \mathscr{b}_{\odot}$ the lifetime of the ultraluminous phase can in principle be well over $10^{9} \mathrm{yr}$, since this process is much more efficient at generating the required energies. However, it is also true that the lifetime of the AGN as a power source can be longer than, or turn on after, the ultraluminous phase itself, since galactic winds can effectively sweep away much of the dust needed to reprocess the emitted energy into the far-infrared. These winds may also reduce the AGN fuel supply, but since the starburst/wind injection region is expected to be on the kpc scale, some material may remain bound to the black hole. The time scale required for a 
black hole radiating at $L=10^{12} L_{\odot}=L$ (Eddington) to increase its mass by a factor of $e$, is only about $4 \times 10^{7} \mathrm{yr}$ (Osterbrock 1993). A black hole radiating at $10 \%$ of the Eddington luminosity would require an order of magnitude longer time to increase its mass by the same fraction. The latter is comparable to the infrared luminous phase lifetimes calculated in the present paper when all the apparently single nucleus galaxies are included in the estimate.

Comparison of the space density of ULIRGs with that of optically selected quasars yields a comparison of the lifetimes of these objects, if we adopt the viewpoint that ULIRGs are the progenitors of quasars. Soifer et al. (1986) report that there are roughly 3.5 times as many ULIRGs as quasars in the luminosity range $\log \left(L_{\mathrm{bol}} / L_{\odot}\right)=12.0-12.5$, making it possible that the ULIRGs can evolve into optical quasars if the ULIRG lifetime is longer than the quasar lifetime. We can now perform this calculation again with a much larger sample of ULIRGs, complete within a larger volume of space. Examination of the $60 \mu \mathrm{m}$ flux density as a function of redshift (or more crudely, the distribution of ULIRGs versus redshift) shows that the 2 Jy ULIRG sample is complete to $\sim 30000 \mathrm{~km} \mathrm{~s}^{-1}$. The space density of quasars in the same volume of space can be inferred from the Bright Quasar Sample (BQS) of Schmidt \& Green (1983). This sample contains 114 quasars covering one quarter of the sky. Using a bolometric luminosity, $L_{\text {bol }}=16.5 \times \nu L_{\nu}(0.43 \mu \mathrm{m})$ (Sanders et al. 1989), and a limiting magnitude of $m_{B}(\mathrm{lim})=15.61$, one finds the quasars with $L_{\text {bol }} \geqslant 8.5 \times 10^{11} L_{\odot}$ (the lower limit of ULIRG luminosities) complete out to $\mathrm{cz}=30000$ $\mathrm{km} \mathrm{s}^{-1}$. We therefore adopt completeness in both the $2 \mathrm{Jy}$ ULIRG and BQS quasar samples out to $30000 \mathrm{~km} \mathrm{~s}^{-1}$. There are 35 ULIRGs and $15 \mathrm{BQS}$ quasars with a bolometric luminosity greater than $8.5 \times 10^{11} L_{\odot}$ within this redshift limit. The area of sky covered in the 2 Jy ULIRG sample is 2.9 times larger than the BQS sample area, leading us to conclude that there are 1.2 times as many quasars as ULIRGs out to $\mathrm{cz}=30000 \mathrm{~km} \mathrm{~s}^{-1}$. If all ultraluminous infrared galaxies evolve into optical quasars then these number densities suggest the lifetimes of the infrared and ultraviolet bright phases of these objects are comparable. This in fact seems quite plausible since the ULIRG lifetime of $2 \times 10^{8} \mathrm{yr}$ calculated in Sec. 4.2 is remarkably close to that calculated above for a quasar radiating at $10 \%$ of its Eddington luminosity.

Removal of the dust that surrounds the source of power in the ULIRGs can proceed via the action of outflowing superwinds, known to exist in some infrared luminous galaxies (Heckman et al. 1990). Estimates of the dynamical time scales of the outflowing winds in nearby, luminous infrared galaxies by Heckman et al. range from a few $\times 10^{7}$ to $10^{8} \mathrm{yr}$. These winds may be critical in transforming a ULIRG into an optically recognizable AGN, and may in some cases shorten the lifetime of the infrared phase beyond merger, since, as is the case in Arp 220, they probably "turn on" well before the merger is complete.

As higher-resolution studies uncover more double nucleus systems among the $2 \mathrm{Jy}$ sample, the estimate of the average lifetime from the two methods above will converge-likely to a few $\times 10^{8} \mathrm{yr}$. The large separation systems detected here may either contain unresolved third nuclei, or be galaxies which have become extremely luminous during the early stages of an interaction. If the former statement is correct, higher-resolution observations may reveal the hidden nuclei. If the latter is correct, recent models will need to be revised to account for early triggering of the ultraluminous phase during the merger of gas-rich galaxies.

\section{SUMMARY}

We present near-infrared and visual images for 46 ultraluminous infrared galaxies. Analysis of these data, together with published data on the 10 ultraluminous infrared galaxies found in the BGS, leads to the following results:

(1) Fifty-three of the 56 ultraluminous galaxies (95\%) appear to be part of interacting or merging systems. These galaxies display a large variety of visual morphologies, ranging from single highly distorted galactic systems to systems containing double nuclei, as well as pairs of interacting spirals connected by luminous bridges and possessing long tidal tails.

(2) Twenty-five of the 53 interacting galaxies (47\%) have apparent double nuclei. Among these 25 galaxies there is a large range in projected nuclear separation, from 0.3 to 48 kpc. There are seven galaxies with projected nuclear separations greater than $10 \mathrm{kpc}$. These large separation interacting pairs may imply that the ultraluminous infrared phase can, in some cases, start early during the interaction when the galaxies are still on highly elliptical orbits. Alternatively, these systems may contain hidden third nuclei. Twenty-eight additional galaxies have distorted morphologies suggesting a strong interaction yet they apparently possess only a single nucleus. The predominance of close double systems and apparently single nucleus galaxies implies that ultraluminous infrared activity strongly favors selection of galaxies with very small physical separations.

(3) Considering only those 53 systems with morphological signs of a current or past interaction, we estimate that the average lifetime of the ultraluminous infrared phase lies between $2 \times 10^{8}$ and $20 \times 10^{8} \mathrm{yr}$. Comparison of these dynamical estimates to models of the merger of gas-rich galaxies and the rates at which fuel is exhausted by either starbursts or the presence of an AGN suggests that the lifetime may be much closer to the smaller of these two values, namely, a few $\times 10^{8} \mathrm{yr}$.

We thank the night assistants at the Palomar Observatory, J. Carasco, S. Staples, and J. Moriarity, and the entire staff at the observatory for their assistance in obtaining these data. Discussions with J. C. Mihos and E. S. Phinney were most helpful. Infrared astronomy at Caltech is supported by grants from NASA and the NSF. This research has made use of the NASA/IPAC Extragalactic Database which is operated by the Jet Propulsion Laboratory, Caltech, under contract with NASA.

\section{APPENDIX A. MORPHOLOGICAL DETAILS}

While many of the galaxies discussed in this paper have morphologies which are easily classifiable (widely separated pairs, single distorted systems with two bright nuclei) some 
are more difficult to interpret. Here, we present a more detailed discussion of the morphologies of these galaxies, and also address some of the more general points relevant to this classification process.

IRAS $19458+0944$ possesses what appears to be a second nucleus in the $r$ band image of Fig. 1, but inspection of the $K$ band frame shows this "nucleus" (at P.A. $=130^{\circ}$, and a distance of $4.5 \mathrm{arcsec}$ ) to be very faint. The flux ratio between this second peak and the primary nucleus is about $27: 1$. If the $K$ band flux from the primary nucleus is dominated by starlight it is unlikely that the second peak represents the nucleus of a companion galaxy since the flux ratio then implies a large mass ratio between the merging galaxy nuclei. Although large flux ratios are possible when one of the nuclei becomes active (e.g., see recent work on Mrk 231 by Armus et al. 1994), the fact that the second peak becomes less prominent at $K$ than it is at $r$ suggests that it may be a star forming knot in the tail of the merger remnant. Given the very crowded field it is also possible that the second peak may be a foreground star.

There are some ULIRGs with nearby stars which, when displayed in contour form, can often appear as companion galaxies. Examples of these are: IRAS 00262+4251, IRAS $03158+4227$, IRAS $05246+0103$, IRAS $08030+5243$, and IRAS 08311-2459. In general, with the large number of stars visible on a single $6.3 \times 6.3$ square arcmin $r$ band frame it is straightforward to exclude nearby, truly point-like objects from being close, interacting galaxies on the basis of the PSF for each image.

IRAS 00456-2904 has (in the $r$ band image of Fig. 1) a bright central galaxy and a faint, resolved object to the northeast. This object does not have a well-defined peak, and is unlikely to be the nucleus of a companion galaxy. We interpret this feature as a relative brightness enhancement in a faint tidal tail emanating from the primary galaxy since the faint emission to the east of the primary points toward this feature. Clearly, deeper $r$ band images, as well as a nearinfrared image of the entire system would help elucidate the true state of this galaxy.

IRAS $10565+2448$ is an interesting example of a possible triple galaxy system. Visual spectra of the two brightest objects in the $r$ band image of Fig. 1 indicate that both are at the same redshift, with the brighter of the two showing strong emission lines, and the fainter member showing strong Balmer absorption lines (Strauss et al. 1995). The nature of the third object located $7.9 \operatorname{arcsec}(6.2 \mathrm{kpc})$ to the southeast of the brightest nucleus is unknown. It is unlikely to be a galactic nucleus, as its $K$ band flux is $\sim 50$ times fainter than that of the primary nucleus. We have therefore assumed that the interaction responsible for triggering the ultraluminous phase is between the large, bright galaxies, and thus use the separation of these nuclei in the ultraluminous phase lifetime calculations.

IRAS $15001+1433$ is a single, distorted galaxy with two, fainter, apparently disconnected knots to the west. The brighter of these knots is 2.3 mag fainter in $r$ than the primary galaxy ( -22.3 vs $-20.0 \mathrm{mag}$, respectively). Although deeper visual images of this system may provide a clear morphological link between the brightest of these knots and the primary galaxy, we have classified this object as a distorted single nucleus system. If the brighter knot to the west is the stripped nucleus of the intruder galaxy, the current separation is at least $37 \mathrm{kpc}$, providing another case for large physical separation pairs. However, due to the lack of a morphological connection, and in light of the disparate magnitudes, we treat this system as an unresolved single nucleus galaxy in the analysis.

IRAS $18470+3233$ may be another triple galaxy system similar to IRAS $10565+2448$. Here again however, the nature of the third object is unknown, and we have thus adopted the separation of the two brightest components (P.A. $\left.=60^{\circ}\right)$ in the merger rate calculations of Sec. 4 .

\section{REFERENCES}

Armus, L., Heckman, T., \& Miley, G. 1987, AJ, 94, 831

Armus, L., Heckman, T., \& Miley, G. 1990, ApJ, 364, 471

Armus, L., Surace, J. A., Soifer, B. T., Matthews, K., Graham, J. R., \&

Larkin, J. E. 1994, AJ, 108, 76

Barnes, J. E., \& Hernquist, L. 1992, ARA\&A, 30, 705

Binney, J., \& Tremaine, S. 1987, in Galactic Dynamics, edited by J. P Ostriker (Princeton University Press, Princeton)

Burstein, D., \& Heiles, C. 1982, AJ, 87, 1165

Carico, D. P., Graham, J. R., Matthews, K., Wilson, T. D., Soifer, B. T., Neugebauer, G., \& Sanders, D. B. 1990, ApJ, 349, L39

Condon, J. J., Huang, Z.-P., \& Yin, Q. F. 1991 ApJ, 378, 65

Cutri, R. M., \& McAlary, C. W. 1985, ApJ, 296, 90

Feigelson, E. D., \& Nelson, P. I. 1985, ApJ, 293, 192

Graham, J. R., Carico, D. P., Matthews, K., Neugebauer, G., Soifer, B. T., \&

Wilson, T. D. 1990, ApJ, 354, L5

Hayes, D. S., \& Latham, D. W. 1975, ApJ, 197, 593

Heckman, T. M., Armus, L., \& Miley, G. K. 1990, ApJS, 74, 833

Heckman, T. M., Lehnert, M. D., \& Armus, L. 1993, in The Environment and Evolution of Galaxies, edited by J. M. Shull and H. A. Thronson, Jr. (Kluwer, Dordrecht), p. 455

IRAS Point Source Catalog Version 2. 1988, Joint IRAS Science Working Group (U.S. GPO, Washington D.C.) (PSC)
Joseph, R. D., \& Wright, G. S. 1984, Nature, 311, 132

Kent, S. M. 1985, PASP, 97, 165

Kirshner, R. P., Oemler, Jr., A., \& Schechter, P. L. 1978, AJ, 83, 1549

Kirshner, R. P., Oemler, Jr., A., \& Schechter, P. L. 1979, AJ, 84, 951

Larkin, J. E., Armus, L., Knop, R. A., Matthews, K., \& Soifer, B. T. 1995, ApJ, 452 (in press)

Lawrence, A., Rowan-Robinson, M., Leech, K., Jones, D. H. P., \& Wall, J. V. 1989, MNRAS, 240, 329

Lonsdale, C. J., Persson, S. E., \& Matthews, K. 1984, ApJ, 287, 95

Majewski, S. R., Hereld, M., Koo, D. C., Illingworth, G. D., \& Heckman, T. M. 1993, ApJ, 402, 125

Mihos, J. C., \& Hernquist, L. 1994, ApJ, 431, L9

Osterbrock, D. E. 1993, ApJ, 404, 551

Rieke, G. H., Cutri, R. M., Black, J. H., Kailey W. F., McAlary, C. W., Lebofsky, M. J., \& Elston, R. 1985, ApJ, 290, 116

Sanders, D. B., Soifer, B. T., Elias, J. H., Madore, B. F., Matthews, K., Neugebauer, G., \& Scoville, N. Z. 1988a, ApJ, 325, 74

Sanders, D. B., Soifer, B. T., Elias, J. H., Neugebauer, G., \& Matthews, K. 1988b, ApJ, 328, L35

Sanders, D. B., Phinney, E. S., Neugebauer, G., Soifer, B. T., \& Matthews, K. 1989, ApJ, 347, 29

Sanders, D. B., Scoville, N. Z., \& Soifer, B. T. 1991, ApJ, 370, 158

Schechter, P. 1976, ApJ, 203, 297 
Schmidt, M., \& Green, R. F. 1983, ApJ, 269, 352

Sebok, W. L. 1986, ApJS, 62, 301

Soifer, B. T., et al. 1984, ApJ, 278, L71

Soifer, B. T., Sanders, D. B., Neugebauer, G., Danielson, G. E., Lonsdale, C.

J., Madore, B. F., \& Persson, S. E. 1986, ApJ, 303, L41

Soifer, B. T., Sanders, D. B., Madore, B. F., Neugebauer, G., Danielson, G.

E., Elias, J. H., Lonsdale, C. J., \& Rice, W. L. 1987, ApJ, 320, 238
Soifer, B. T., Boehmer, L., Neugebauer, G, \& Sanders, D. B. 1989, AJ, 98, 766

Strauss, M. A., Davis, M., Yahil, A., \& Huchra, J. P. 1990, ApJ, 361, 49

Strauss, M. A., Huchra, J. P., Davis, M., Yahil, A., Fisher, K. B., \& Tonry, J. 1992, ApJS, 83, 29

Strauss, M. A., et al. 1995, in preparation

Thuan, T. X., \& Gunn, J. E. 1976, PASP, 88, 543 